\title{
Light-cone modular bootstrap and pure gravity
}

\author{
Nathan Benjamin $\odot,{ }^{1, *}$ Hirosi Ooguri, ${ }^{2,3, \dagger}$ Shu-Heng Shao ${ }^{4, *}$ and Yifan Wang ${ }^{5, \S}$ \\ ${ }^{1}$ Princeton Center for Theoretical Science, Princeton University, Princeton, New Jersey 08544, USA \\ ${ }^{2}$ Walter Burke Institute for Theoretical Physics, California Institute of Technology, \\ Pasadena, California 91125, USA \\ ${ }^{3}$ Kavli Institute for the Physics and Mathematics of the Universe (WPI), University of Tokyo, \\ Kashiwa, 277-8583, Japan \\ ${ }^{4}$ School of Natural Sciences, Institute for Advanced Study, Princeton, New Jersey 08540, USA \\ ${ }^{5}$ Joseph Henry Laboratories, Princeton University, Princeton, New Jersey 08544, USA
}

(Received 7 July 2019; published 23 September 2019)

\begin{abstract}
We explore the large spin spectrum in two-dimensional conformal field theories with a finite twist gap, using the modular bootstrap in the light-cone limit. By recursively solving the modular crossing equations associated with different $\operatorname{PSL}(2, \mathbb{Z})$ elements, we identify the universal contribution to the density of large spin states from the vacuum in the dual channel. Our result takes the form of a sum over $\operatorname{PSL}(2, \mathbb{Z})$ elements, whose leading term generalizes the usual Cardy formula to a wider regime. Rather curiously, the contribution to the density of states from the vacuum becomes negative in a specific limit, which can be canceled by that from a nonvacuum Virasoro primary whose twist is no bigger than $\frac{c-1}{16}$. This suggests a new upper bound of $\frac{c-1}{16}$ on the twist gap in any $c>1$ compact, unitary conformal field theory with a vacuum, which would in particular imply that pure $\mathrm{AdS}_{3}$ gravity does not exist. We confirm this negative density of states in the pure gravity partition function by Maloney, Witten, and Keller. We generalize our discussion to theories with $\mathcal{N}=(1,1)$ supersymmetry and find similar results.
\end{abstract}

DOI: 10.1103/PhysRevD.100.066029

\section{INTRODUCTION}

Despite progress in the classification program of rational conformal field theories, we have shockingly little understanding of the general landscape of two-dimensional (2D) conformal field theories (CFTs). For example, there is no explicit construction of any 2D compact, unitary CFT with central charge $c>1$ and no Virasoro conserved currents of any spin [1]. Such CFTs are expected to be generic, and our ignorance of them clearly shows the limitation in our understanding.

To go beyond the realm of rational CFTs, we will consider CFTs with a finite twist gap. The twist of an operator is defined as $\Delta-|j|=2 \min (h, \bar{h})$. Theories with conserved currents, such as rational CFTs, necessarily have

\footnotetext{
"nathanb@princeton.edu

ooguri@caltech.edu

shao@ias.edu

§yifanw@princeton.edu
}

Published by the American Physical Society under the terms of the Creative Commons Attribution 4.0 International license. Further distribution of this work must maintain attribution to the author(s) and the published article's title, journal citation, and DOI. Funded by SCOAP. a vanishing twist gap. ${ }^{1}$ For this reason we will think of the twist gap as a measure on how irrational a CFT is.

In this paper, we will address the following two general questions for CFTs with a finite twist gap:

(1) Is there a universal Cardy-like growth for the large spin states?

(2) At a fixed central charge $c$, how large can the twist gap be?

These two questions are tied together by modular invariance of the torus partition function.

We start with the first question. We generalize Cardy's argument for the asymptotic growth of states with large scaling dimensions [2]. More specifically, we analytically continue the torus moduli $\tau, \bar{\tau}$ to two independent complex variables, and consider the limit $\operatorname{Im}(\tau) \rightarrow 0$ while keeping $\bar{\tau}$ fixed. This is similar to the light-cone limit studied in the conformal bootstrap of four-point functions [3,4], but now applied to the modular bootstrap program [5]. By solving the modular crossing equations in the light-cone limit with arbitrary rational real parts of $\tau, \bar{\tau}^{2}$ we recursively identify

\footnotetext{
${ }^{1}$ A priori, there could be theories without conserved currents, but with an accumulation of operators toward vanishing twist, and therefore have zero twist gap.

${ }^{2}$ These are the $\operatorname{PSL}(2, \mathbb{Z})$ images of the cusp at $\tau=i \infty$.
} 
the universal contribution from the Virasoro vacuum multiplet to the density of large spin states for any $c>1$ CFT with a finite twist gap. Our formula generalizes the usual Cardy formula from the regime $h, \bar{h} \gg c$ to $h \gg c$ but with $\bar{h}-$ $\frac{c-1}{24}>0$ finite. The universal density of states takes the form of a sum over $\operatorname{PSL}(2, \mathbb{Z})$ images, ${ }^{3}$ whose leading term is the extended Cardy formula discussed recently in [6-8]. The density of states depends on the number-theoretic properties of the spin $j=h-\bar{h}$ and is in particular nonanalytic in $j$.

Now we turn to the second question. Rather curiously, our density of states from the vacuum contribution becomes negative in the double limit where $j \rightarrow \infty$ and $\bar{h}-\frac{c-1}{24} \rightarrow 0$. Such negative density of states of course should not be present in a physical, unitary CFT. This negativity can be canceled by the contribution from a nonvacuum primary operator of twist $\Delta-|j|$ at or below $\frac{c-1}{16}$ in the dual channel. We are therefore led to the following tentative conclusion: any compact, unitary CFT with a $\operatorname{PSL}(2, \mathbb{C})$ invariant vacuum must have a twist gap of at most $\frac{c-1}{16}$. Our argument is not yet rigorous, and we will discuss the gaps to complete the proof. If true, our result improves the earlier $\frac{c-1}{12}$ bound on the twist gap by Hartman and [1]. ${ }^{4}$

Via the holographic correspondence [9], our result has interesting implications on pure Einstein gravity as a quantum gravity theory in $\mathrm{AdS}_{3}$. In the strictest sense, pure $\mathrm{AdS}_{3}$ gravity is dual to a $2 \mathrm{D}$ large $c$, unitary CFT where all nonvacuum Virasoro primary operators have $h, \bar{h} \geq \frac{c-1}{24}$ and are interpreted as Bañados-TeitelboimZanelli (BTZ) black holes. The new twist gap bound $\frac{c-1}{16}$ suggested by our argument would imply that pure $\mathrm{AdS}_{3}$ gravity does not exist. ${ }^{5}$ Indeed, we will check explicitly that the pure gravity partition function computed by MaloneyWitten-Keller [12,13] agrees with our formula in the specific double limit mentioned above. The sum over the $P S L(2, \mathbb{Z})$ elements in our formula is identified as a sum over geometries in $\mathrm{AdS}_{3} .{ }^{6}$ In particular, we confirm that the pure gravity partition function has an identical negative density of states in this limit. This gives another interpretation of our result: while the pure gravity partition function

\footnotetext{
${ }^{3}$ More precisely, this is a sum over the coset $\operatorname{PSL}(2, \mathbb{Z}) / \Gamma_{\infty}$ where $\Gamma_{\infty}$ is the subgroup generated by $T: \tau \rightarrow \tau+1$ that stabilizes the cusp at $\tau=i \infty$.

${ }^{4}$ In [1] this argument was credited to Tom Hartman.

${ }^{5}$ Recently, the closest theory to pure gravity in $\mathrm{AdS}_{2}$, the Jackiw-Teitelboim theory, has been shown to be dual to a random matrix model, rather than a single quantum system with a definite Hamiltonian [10]. Furthermore, a pure AdS gravity theory, if it exists, would have been a counterexample to the swampland conjecture in [11]. The current paper provides another piece of evidence that pure gravity in $\mathrm{AdS}_{3}$ might not be dual to a single unitary 2D CFT.

${ }^{6} \mathrm{~A}$ similar sum over $\operatorname{PSL}(2, \mathbb{Z})$ images was originally interpreted as a sum over gravitational saddle points in [14] for elliptic genera. Each term in the $\operatorname{PSL}(2, \mathbb{Z})$ sum corresponds to a different $\mathrm{AdS}_{3}$ geometry discussed in [15].
}

of $[12,13]$ is unphysical in various ways, it approximates the universal density of large spin states dictated by the vacuum state in the dual channel of the modular crossing equation. In other words, the pure gravity partition function of Maloney-Witten-Keller is the analog of double-twist operators in $d>2[3,4]$, or of "Virasoro mean field theory" in 2D [7] for the modular bootstrap (see also [16]).

The paper is organized as follows. In Sec. II we review the argument by Hartman and [1] for the $\frac{c-1}{12}$ bound on the twist gap. In Sec. III, the extended Cardy formula for the density of large spin states is reviewed. In Sec. IV, we generalize the extended Cardy formula to include subleading corrections by solving recursively the crossing equations associated with general elements of $\operatorname{PSL}(2, \mathbb{Z})$. In Sec. V, the implications of this universal density of large spin states are discussed, which suggest that the twist gap in any compact, unitary $c>1 \mathrm{CFT}$ can be at most $\frac{c-1}{16}$. Section VI discusses the interpretation of our result in relation to the pure $\mathrm{AdS}_{3}$ gravity partition function. In Sec. VII, we discuss the $\mathcal{N}=(1,1)$ supersymmetric generalization. The Appendixes A and B describe some technical steps needed in solving the crossing equations. Appendix $\mathrm{C}$ discusses some subtleties present when there is an accumulation of operators in twist. In Appendix D, we record the modular crossing kernels for more general elements of $\operatorname{PSL}(2, \mathbb{Z})$.

\section{WARM-UP: THE $\frac{c-1}{12}$ TWIST GAP}

In this section we review an argument by Hartman and [1] showing that the twist gap in any compact unitary $2 \mathrm{D}$ CFT has to be no larger than $\frac{c-1}{12}$. This argument has been generalized from the Virasoro algebra to the $\mathcal{W}_{N}$ algebra in [17].

Consider the partition function $Z(q, \bar{q})$ of a 2D CFT on a torus with complex structure moduli $q=\exp (2 \pi i \tau)$, $\bar{q}=\exp (-2 \pi i \bar{\tau})$. We will analytically continue so that $\tau$ and $\bar{\tau}$ are two independent complex variables. Let us parametrize the torus moduli as

$$
\tau=i \frac{\beta}{2 \pi}, \quad \bar{\tau}=-i \frac{\bar{\beta}}{2 \pi},
$$

and take $\beta, \bar{\beta}$ to be independent positive numbers. The physical interpretation of this analytic continuation of $\tau, \bar{\tau}$ to two independent imaginary values is the following. The torus partition function can be interpreted as the twist-field four-point function in the symmetric product of two identical copies of the original CFT. Then taking $\tau, \bar{\tau}$ independently to be purely imaginary corresponds to the Lorentzian regime of the twist-field four-point function. Later we will take $\beta \rightarrow 0$ while keeping $\bar{\beta}$ fixed, which is the light-cone limit from the twist-field four-point function point of view. We have

$$
q=\exp (-\beta), \quad \bar{q}=\exp (-\bar{\beta})
$$


Their modular $S$ transforms will be denoted with a prime:

$$
q^{\prime}=\exp \left(-\frac{4 \pi^{2}}{\beta}\right), \quad \bar{q}^{\prime}=\exp \left(-\frac{4 \pi^{2}}{\bar{\beta}}\right) .
$$

The torus partition function can be expanded in Virasoro characters. For a 2D unitary CFT with $c>1$, the possible modules of the Virasoro algebra are the degenerate module, i.e., the vacuum module $h=0$, and a continuous family of nondegenerate modules labeled by a positive conformal weight $h>0$. Their Virasoro characters are given by

$$
\chi_{0}(q)=(1-q) \frac{q^{-\frac{c-1}{24}}}{\eta(q)}, \quad \chi_{h>0}(q)=\frac{q^{h-\frac{c-1}{24}}}{\eta(q)} .
$$

Combining the left with the right, there are three kinds of Virasoro primaries:

$$
\text { (vacuum) } \chi_{0}(q) \chi_{0}(\bar{q}),
$$

(conserved current) $\quad \chi_{0}(q) \chi_{\bar{h}>0}(\bar{q}), \quad \chi_{h>0}(q) \chi_{0}(\bar{q})$,

$$
\text { (nondegenerate) } \chi_{h>0}(q) \chi_{\bar{h}>0}(\bar{q}) \text {. }
$$

Consider an operator with conformal weights $(h, \bar{h})$. The scaling dimension $\Delta$ and the spin $j$ are defined as

$$
\Delta=h+\bar{h}, \quad j=h-\bar{h} .
$$

The twist of an operator is defined as $\Delta-|j|$, and we will denote half of the twist as $t$ :

$$
t \equiv \min (h, \bar{h})=\frac{\Delta-|j|}{2} .
$$

We would like to study 2D CFTs with the finite twist gap $2 t_{\text {gap }}>0$. In particular, this implies that there is no conserved current in the theory. Under the finite twist gap assumption, the torus partition function can be expanded as

$$
\begin{aligned}
Z(q, \bar{q}) & =\chi_{0}(q) \chi_{0}(\bar{q})+\sum_{h, \bar{h} \geq t_{\text {gap }}} n_{h, \bar{h}} \chi_{h}(q) \bar{\chi}_{\bar{h}}(\bar{q}) \\
& =\sum_{j \in \mathbb{Z}} \int_{0}^{\infty} d t \rho_{j}(t) \chi_{h}(q) \bar{\chi}_{\bar{h}}(\bar{q}),
\end{aligned}
$$

where $n_{h, \bar{h}} \in \mathbb{N}$ is the number of Virasoro primaries with conformal weights $(h, \bar{h})$. In the second line we have introduced the density of Virasoro primaries $\rho_{j}(t)$, defined as a discrete sum over delta functions:

$$
\rho_{j}(t) \equiv \sum_{\mathcal{O} \text { with spin } j} \delta\left(t-t_{\mathcal{O}}\right),
$$

where the sum is over the Virasoro primaries in the spectrum with $\operatorname{spin} j$, and $t_{\mathcal{O}}$ refers to $\min \left(h_{\mathcal{O}}, \bar{h}_{\mathcal{O}}\right)$. In what follows we will refer to $\rho_{j}(t)$ as the "density of states" even though it is really the density of Virasoro primaries.

Using modular invariance $Z(q, \bar{q})=Z\left(q^{\prime}, \bar{q}^{\prime}\right)$, we can rewrite the partition function in the dual channel:

$$
\begin{aligned}
Z\left(q^{\prime}, \bar{q}^{\prime}\right)= & \sum_{j^{\prime} \in \mathbb{Z}} \int_{0}^{\infty} d t^{\prime} \rho_{j^{\prime}}\left(t^{\prime}\right) \chi_{h^{\prime}}\left(q^{\prime}\right) \bar{\chi}_{\bar{h}^{\prime}}\left(\bar{q}^{\prime}\right) \\
= & \frac{\exp \left(\frac{4 \pi^{2}}{\beta} \frac{c-1}{24}\right)}{\eta\left(q^{\prime}\right)}\left[\left(1-e^{-4 \pi^{2} / \beta}\right) \chi_{0}\left(\bar{q}^{\prime}\right)\right. \\
& \left.+\sum_{j \in \mathbb{Z}} \int_{t_{\mathrm{gap}}}^{\infty} d t^{\prime} \rho_{j^{\prime}}\left(t^{\prime}\right) e^{-4 \pi^{2} h^{\prime} / \beta} \chi_{\bar{h}^{\prime}}\left(\bar{q}^{\prime}\right)\right] .
\end{aligned}
$$

Up to this point all the equations are exact with no approximation. We now equate (2.8) with (2.10) and take the $\beta \rightarrow 0$ limit:

$$
\begin{aligned}
& \operatorname{vac}+\sum_{j \in \mathbb{Z}} \int_{t_{\text {gap }}}^{\infty} d t \rho_{j}(t) e^{-\beta\left(h-\frac{c-1}{24}\right)} e^{-\bar{\beta}\left(\bar{h}-\frac{c-1}{24}\right)} \\
& =\sqrt{\frac{4 \pi^{2}}{\beta \bar{\beta}}} e^{\frac{4 \pi^{2} c-1}{\beta} 24} e^{\frac{4 \pi^{2} c-1}{\bar{\beta} 24}}\left[\left(1-e^{-\frac{4 \pi^{2}}{\beta}}\right)\left(1-e^{-\frac{4 \pi^{2}}{\bar{\beta}}}\right)+\cdots\right],
\end{aligned}
$$

where vac $\equiv(1-q)(1-\bar{q}) q^{-\frac{c-1}{24}} \bar{q}^{-\frac{c-1}{24}}$. We have used $\eta\left(q^{\prime}\right)=\sqrt{\frac{\beta}{2 \pi}} \eta(q)$. The $\cdots$ are contributions from the nonvacuum operators in the cross channel. As we take $\beta \rightarrow 0$ (but keep $\bar{\beta}$ finite), the divergence on the right-hand side (RHS) has to be reproduced by an infinite number of states on the left-hand side (LHS).

Let us further simplify the LHS of (2.11) in the $\beta \rightarrow 0$ limit. First we can drop the vacuum term since any individual term does not give a divergence as $\beta \rightarrow 0$. We then write the LHS as

$$
\begin{aligned}
& \sum_{j=0}^{\infty} e^{-\beta j} \int_{t_{\mathrm{gap}}}^{\infty} d \bar{h} \rho_{j}(\bar{h}) \exp \left[-(\beta+\bar{\beta})\left(\bar{h}-\frac{c-1}{24}\right)\right] \\
& +\sum_{j=-\infty}^{-1} e^{\bar{\beta} j} \int_{t_{\mathrm{gap}}}^{\infty} d h \rho_{j}(h) \exp \left[-(\beta+\bar{\beta})\left(h-\frac{c-1}{24}\right)\right] .
\end{aligned}
$$

Note that $t=\bar{h}$ if $j \geq 0$ and $t=h$ if $j<0$. In the $\beta \rightarrow 0$ limit (while keeping $\bar{\beta}$ finite), the second term is finite, which can thus be ignored. Moreover, we can replace $\beta+\bar{\beta}$ in the first term by $\bar{\beta}$ in this limit. Hence, Eq. (2.11) in the $\beta \rightarrow 0$ limit becomes 


$$
\begin{aligned}
\sum_{j=0}^{\infty} & e^{-\beta j} \int_{t_{\text {gap }}}^{\infty} d \bar{h} \rho_{j}(\bar{h}) \exp \left[-\bar{\beta}\left(\bar{h}-\frac{c-1}{24}\right)\right] \\
= & \frac{2 \pi}{\sqrt{\beta \bar{\beta}}} e^{\frac{4 \pi^{2} c-1}{\beta} 24} e^{\frac{4 \pi^{2} c-1}{\beta} 24}\left(1-e^{-\frac{4 \pi^{2}}{\beta}}\right)\left(1-e^{-\frac{4 \pi^{2}}{\beta}}\right) \\
& +\mathcal{O}\left(e^{\frac{4 \pi^{2}}{\beta}\left(\frac{c-1}{24}-t_{\text {gap }}\right)}\right) .
\end{aligned}
$$

Using (2.13), we now prove the twist gap $2 t_{\text {gap }}$ cannot be larger than $\frac{c-1}{12}$. Let us assume otherwise, i.e., $2 t_{\text {gap }}>\frac{c-1}{12}$. We multiply both sides by $e^{\bar{\beta}\left(t_{\text {gap }}-\frac{c-1}{24}\right)}$. Then the LHS has a negative $\bar{\beta}$ derivative, but the $\bar{\beta}$ derivative of the RHS will eventually be positive for large enough $\bar{\beta}$ (while still keeping $\bar{\beta} \ll 1 / \beta$ ) due to the exponential growth of the factor $e^{\bar{\beta}\left(t_{\text {gap }}-\frac{c-1}{24}\right)}$. We therefore arrive at a contradiction.

\section{EXTENDED CARDY FORMULA}

In this section, we will review the derivation in [6-8] of the universal spectrum of large spin Virasoro primaries for all $c>1$ 2D CFTs with nonzero twist gap, i.e., $2 t_{\text {gap }}>0$. We will argue that the physical density of states $\rho_{j}(\bar{h})$ in the large spin $j \gg c$ limit is universally approximated by

$$
\begin{aligned}
\rho_{j, 1}^{0}(\bar{h})= & \frac{e^{4 \pi \sqrt{(\bar{h}+j)\left(\frac{c-1}{24}\right)}}}{\sqrt{\left(\bar{h}+j-\frac{c-1}{24}\right)\left(\bar{h}-\frac{c-1}{24}\right)}} \theta\left(\bar{h}-\frac{c-1}{24}\right) \\
& \times\left[\cosh \left(4 \pi \sqrt{\left(\frac{c-1}{24}\right)\left(\bar{h}-\frac{c-1}{24}\right)}\right)\right. \\
& \left.-\cosh \left(4 \pi \sqrt{\left(\frac{c-25}{24}\right)\left(\bar{h}-\frac{c-1}{24}\right)}\right)\right] .
\end{aligned}
$$

We use the superscript 0 to remind the reader that, much as the usual Cardy formula, $\rho_{j, 1}^{0}(t)$ is a continuous function of the twist $2 t$ that at large spin approximates the physical density of states $\rho_{j}(t)$, which is a sum of delta functions. The meaning of the subscript 1 , on the other hand, will become clear in Sec. IV. Here $\theta(x)$ is the Heaviside step function that equals 1 if $x>0$ and 0 otherwise. Note that the twist gap for this solution is $2 t_{\text {gap }}=\frac{c-1}{12}$. In the limit $j \gg c$ with $\bar{h}>\frac{c-1}{24}$, this can be written more compactly as

$$
\rho_{j, 1}^{0}(\bar{h})=K_{S}(\bar{h}+j) K_{S}(\bar{h}),
$$

where $K_{S}(\bar{h})$ is the modular kernel for the $S$ transformation [18]

$$
\begin{aligned}
K_{S}(\bar{h})= & \sqrt{\frac{2}{\bar{h}-\frac{c-1}{24}}}\left[\cosh \left(4 \pi \sqrt{\left(\frac{c-1}{24}\right)\left(\bar{h}-\frac{c-1}{24}\right)}\right)\right. \\
& \left.-\cosh \left(4 \pi \sqrt{\left(\frac{c-25}{24}\right)\left(\bar{h}-\frac{c-1}{24}\right)}\right)\right] .
\end{aligned}
$$

The asymptotic growth (3.1) generalizes the usual Cardy formula [2], which holds without assuming $2 t_{\text {gap }}>0$, beyond its regime of applicability $h, \bar{h} \gg c$. In the $\bar{h} \gg c$ limit of (3.1), $\rho_{j, 1}^{0}(\bar{h})$ reduces to the usual Cardy formula,

$$
\begin{aligned}
\rho_{j, 1}^{0}(\bar{h}) \rightarrow & \frac{1}{2 \sqrt{\left(h-\frac{c-1}{24}\right)\left(\bar{h}-\frac{c-1}{24}\right)}} \\
& \times e^{4 \pi \sqrt{\left(\frac{c-1}{24}\right) h}+4 \pi \sqrt{\left(\frac{c-1}{24}\right) \bar{h}}}, \quad h, \bar{h} \gg c .
\end{aligned}
$$

For this reason, we will refer to (3.1) as the extended Cardy formula.

Before we verify that (3.1) is a solution to our crossing equation (2.13), we first discuss the defining property of the modular kernel $K_{S}(\bar{h})$. It relates the vacuum Virasoro character in one channel to the nondegenerate Virasoro characters in the crossed channel,

$$
\chi_{0}\left(\bar{q}^{\prime}\right)=\int_{\frac{c-1}{24}}^{\infty} d \bar{h} K_{S}(\bar{h}) \chi_{\bar{h}}(\bar{q}),
$$

or equivalently

$$
e^{\frac{4 \pi^{2} c-1}{\bar{\beta} 24}}\left(1-e^{-\frac{4 \pi^{2}}{\bar{\beta}}}\right)=\sqrt{\frac{\bar{\beta}}{2 \pi}} \int_{\frac{c-1}{24}}^{\infty} d \bar{h} K_{S}(\bar{h}) e^{-\bar{\beta}\left(\bar{h}-\frac{c-1}{24}\right)}
$$

and similarly for the holomorphic (left-moving) characters. This equation will be crucial for our crossing solution.

The argument leading to the extended Cardy formula (3.1) is similar to the original argument by Cardy, but now in the light-cone limit where $\beta \rightarrow 0$ while $\bar{\beta}$ is held fixed. This leads to the crossing equation (2.13), where the divergence on the RHS needs to be reproduced by a certain asymptotic growth of states with large spin. Below we show that (3.1) is indeed a solution to the crossing equation. Plugging in the solution (3.1) into (2.13), the LHS becomes

$$
\sum_{j=0}^{\infty} e^{-\beta j} \int_{\frac{c-1}{24}}^{\infty} d \bar{h} K_{S}(\bar{h}+j) K_{S}(\bar{h}) \exp \left[-\bar{\beta}\left(\bar{h}-\frac{c-1}{24}\right)\right] .
$$

In Appendix A, we show that in the small $\beta$ limit, the sum over $j$ in the above equation can be approximated by an integral over $j$, up to a term that is $\beta$ independent, which can be absorbed in the error term of the RHS of (2.13). Shifting integration variables and using (3.6), we then reproduce the 
leading divergent terms in the $\beta \rightarrow 0$ limit on the RHS of (2.13).

Therefore, Eq. (3.1) is indeed a solution to the crossing equation and gives the universal density of large spin states in any compact unitary CFT with a finite twist gap, up to an error that grows slower than $\exp \left(4 \pi \sqrt{\frac{c-1}{24} j}\right)$ in the large spin limit. On the other hand, $\rho_{j, 1}^{0}=0$ if $\bar{h}<\frac{c-1}{24}$, meaning that there is no exponential growth in the large spin limit.

Let us comment on the corrections to (3.1) in the large spin limit. First, there are error terms from approximating the discrete spectrum by a continuous density of states. This error for the density of states $\rho(\Delta)$ that is insensitive to the spin has recently been quantified in $[19,20]$ (see also Appendix C of [21]). Second, there are contributions to the density of states from the lowest twist, nonvacuum primary operators, corresponding to the last term in (2.13). Last, there are contributions coming from the vacuum but for different elements of $\operatorname{PSL}(2, \mathbb{Z})$. The last two corrections will be discussed in later sections.

We emphasize that the converse of our statement here is also true, which follows simply from running our argument backwards. Namely, if a 2D CFT has a large spin spectrum that satisfies (3.1), it is guaranteed to have a nonzero twist gap $2 t_{\text {gap }}>0$. It would be interesting if there is a holographic interpretation of (3.1) for $\bar{h}$ not in the Cardy regime, in terms of the entropy of BTZ black holes. If so, it may suggest the theories holographically dual to Einstein gravity in $\mathrm{AdS}_{3}$ generically have a nonzero twist gap.

\section{IV. $\operatorname{PSL}(2, \mathbb{Z})$ MODULAR CROSSING EQUATIONS}

In this section we will repeat the analyses in Secs. II and III, but with a more general $\operatorname{PSL}(2, \mathbb{Z})$ transformation, and we will find qualitatively new behavior.

\section{A. Crossing Equation}

We now repeat the previous analysis but instead take ${ }^{7}$

$$
\tau=i \frac{\beta}{2 \pi}+\frac{r}{s}, \quad \bar{\tau}=-i \frac{\bar{\beta}}{2 \pi}+\frac{r}{s} .
$$

Here $r$ and $s$ are two coprime integers, with $s$ positive. As before, we consider the limit $\beta \rightarrow 0$ at fixed $\bar{\beta}$. The $q$ variables are

$$
q=\exp \left(-\beta+\frac{2 \pi i r}{s}\right), \quad \bar{q}=\exp \left(-\bar{\beta}-\frac{2 \pi i r}{s}\right) .
$$

Let us act on $\tau, \bar{\tau}$ with the modular transformation

\footnotetext{
${ }^{7}$ Note that there is a slight generalization of this we can consider, in which $\operatorname{Re}(\tau) \neq \operatorname{Re}(\bar{\tau})$. We will not pursue this generalization in the current paper.
}

$$
\left(\begin{array}{cc}
a & b \\
s & -r
\end{array}\right) \in S L(2, \mathbb{Z}), \quad-a r-b s=1,
$$

to get

$$
\begin{aligned}
\tau^{\prime} & =\frac{2 \pi i}{\beta s^{2}}+\frac{a}{s}, \quad \bar{\tau}^{\prime}=-\frac{2 \pi i}{s^{2} \bar{\beta}}+\frac{a}{s} \\
q^{\prime} & =\exp \left(-\frac{4 \pi^{2}}{s^{2} \beta}+2 \pi i \frac{a}{s}\right), \quad \bar{q}^{\prime}=\exp \left(-\frac{4 \pi^{2}}{s^{2} \bar{\beta}}-2 \pi i \frac{a}{s}\right) .
\end{aligned}
$$

Now we use modular invariance of the torus partition function to set (2.8) and (2.10) equal with the new parametrizations of $q$ and $\bar{q}$ as above. Since each individual term in (2.8) is not sufficient to reproduce the divergence in (2.10) as we take $\beta \rightarrow 0$, we can drop the isolated vacuum contribution in (2.8) and obtain

$$
\begin{aligned}
& \sum_{j \in \mathbb{Z}} \int_{t_{\text {gap }}}^{\infty} d t \rho_{j}(t) \exp \left[-\beta\left(h-\frac{c-1}{24}\right)-\bar{\beta}\left(\bar{h}-\frac{c-1}{24}\right)\right. \\
& \left.\quad+2 \pi i \frac{r}{s}(h-\bar{h})\right] \\
& =\frac{2 \pi}{s \sqrt{\beta \bar{\beta}}} e^{\frac{4 \pi^{2}(c-1)}{24 s^{2} \beta}} e^{\frac{4 \pi^{2}(c-1)}{24 s^{2} \bar{\beta}}}\left(1-e^{-\frac{4 \pi^{2}}{s^{2} \beta}+\frac{2 \pi i a}{s}}\right)\left(1-e^{-\frac{4 \pi^{2}-2 \pi i a}{s^{2} \bar{\beta}}-\frac{2 \pi}{s}}\right) \\
& \quad+\mathcal{O}\left(e^{\frac{4 \pi^{2}}{s^{2} \beta}\left(\frac{c-1}{24}-t_{\mathrm{gap}}\right)}\right) .
\end{aligned}
$$

From (4.3), we see that $a r \equiv-1(\bmod s)$. Moreover (4.5) is invariant under $a \rightarrow a+s$. We will henceforth write $a$ as the modular inverse of $-r$ mod $s$, i.e., $a=-\left(r^{-1}\right)_{s}$.

Following the same steps to (2.13), we simplify the above crossing equation in the $\beta \rightarrow 0$ limit to

$$
\begin{aligned}
& \sum_{j=0}^{\infty} \exp \left[-\left(\beta-\frac{2 \pi i r}{s}\right) j\right] \int_{t_{\mathrm{gap}}}^{\infty} d \bar{h} \rho_{j}(\bar{h}) \exp \left[-\bar{\beta}\left(\bar{h}-\frac{c-1}{24}\right)\right] \\
& =\frac{2 \pi}{s \sqrt{\beta \bar{\beta}}} e^{\frac{4 \pi^{2} c-1}{s^{2} \beta^{24}}} e^{\frac{4 \pi^{2} c-1}{s^{2} \bar{\beta} 24}}\left(1-e^{-\frac{4 \pi^{2}}{s^{2} \beta} \frac{2 \pi i\left(r^{-1}\right) s}{s}}\right)\left(1-e^{-\frac{4 \pi^{2}}{s^{2} \bar{\beta}}+\frac{2 \pi i\left(r^{-1}\right) s}{s}}\right) \\
& \quad+\mathcal{O}\left(e^{\frac{4 \pi^{2}}{s^{2} \beta}\left(\frac{c-1}{24}-t_{\text {gap }}\right)}\right) .
\end{aligned}
$$

Note that we reproduce (2.13) if we take $r=0, s=1$. This is our main equation for the modular crossing equations associated with more general $\operatorname{PSL}(2, \mathbb{Z})$ elements.

\section{B. Solution to the crossing equations}

The universal density of states in (3.1) does not successfully reproduce the RHS of (4.6). In particular, if we plug (3.1) into (4.6), the LHS in the $\beta \rightarrow 0$ is finite and fails to provide the divergence $\exp \left(\frac{4 \pi^{2}}{s^{2} \beta} \frac{c-1}{24}\right)$ on the RHS of the equation. See Appendix B for detailed derivations. 
This implies that there must be other universal contributions to the density of large spin states that, while being subleading to (3.1), are responsible for solving the more general crossing equations (4.6).

Our solution to (4.6) will involve the Kloosterman sum defined by

$$
S(j, J ; s)=\sum_{\substack{r: \operatorname{cod}(r, s)=1 \\ 0 \leq r<s}} \exp \left(2 \pi i \frac{r j+\left(r^{-1}\right)_{s} J}{s}\right),
$$

which is sensitive to the number-theoretic properties of the integer spin $j$. Some basic properties of the Kloosterman sum are $S(j, J ; s)=S(-j,-J ; s), S(j, J ; s)=S(J, j ; s)$, and $S(j+s, J ; s)=S(j, J+s ; s)=S(j, J ; s)$. In addition,

$$
\sum_{j=0}^{\operatorname{lcm}\left(s, s^{\prime}\right)-1} S\left(j, J ; s^{\prime}\right) e^{-\frac{2 \pi i r j}{s}}=s e^{\frac{2 \pi i\left(r^{-1}\right) s^{J}}{s}} \delta_{s, s^{\prime}} .
$$

Finally we define the following functions:

$$
\begin{aligned}
d_{0}(h, s)= & \sqrt{\frac{2}{s\left(h-\frac{c-1}{24}\right)}} \cosh \left(\frac{4 \pi}{s} \sqrt{\left(h-\frac{c-1}{24}\right)\left(\frac{c-1}{24}\right)}\right) \\
& \times \theta\left(h-\frac{c-1}{24}\right), \\
d_{1}(h, s)= & \sqrt{\frac{2}{s\left(h-\frac{c-1}{24}\right)}} \cosh \left(\frac{4 \pi}{s} \sqrt{\left(h-\frac{c-1}{24}\right)\left(\frac{c-25}{24}\right)}\right) \\
& \times \theta\left(h-\frac{c-1}{24}\right),
\end{aligned}
$$

and

$$
\begin{aligned}
\rho_{j, s}(h, \bar{h})= & S(j, 0 ; s) d_{0}(h, s) d_{0}(\bar{h}, s) \\
& -S(j,-1 ; s) d_{0}(h, s) d_{1}(\bar{h}, s) \\
& -S(j, 1 ; s) d_{1}(h, s) d_{0}(\bar{h}, s) \\
& +S(j, 0 ; s) d_{1}(h, s) d_{1}(\bar{h}, s) .
\end{aligned}
$$

In the large $j$ limit, the continuous solution to (4.6) is given by $^{8}$

$$
\rho_{j}^{0}(\bar{h})=\sum_{s^{\prime}=1} \rho_{j, s^{\prime}}(\bar{h}+j, \bar{h}), \quad j \gg c .
$$

Importantly, the dependence on the spin $j$ is highly nonanalytic and depends on the number-theoretic property through the Kloosterman sum (4.7). The $s^{\prime}=1$ term is the

\footnotetext{
${ }^{8}$ As in Sec. III, we use the superscript 0 to emphasize this is a continuous function in the twist which approximates the physical density of states $\rho_{j}(t)$. By contrast, the latter is a sum of delta functions.
}

extended Cardy formula (3.1), which grows as $\exp \left(4 \pi \sqrt{\frac{c-1}{24} j}\right)$ in the large spin limit. The higher $s^{\prime}$ terms grow as $\exp \left(\frac{4 \pi}{s^{\prime}} \sqrt{\frac{c-1}{24} j}\right)$ and are subleading corrections to $(3.1)$.

Let us comment on the sum in $s^{\prime}$. The solution presented above is designed to reproduce the divergence on the RHS of the crossing equation (4.6) as $\beta \rightarrow 0$. However, the divergence on the RHS is present only if $s \lesssim 1 / \sqrt{\beta}$. The divergence, if present, controls the density of states whose spins are of the order $j \sim 1 / \beta$ due to the suppression factor $e^{-\beta j}$ on the LHS of (4.6). It follows that for a fixed large spin $j$, we can trust the solution only if $s \lesssim \sqrt{j}$; therefore the sum in (4.11) should be truncated before order $\sqrt{j}$.

For a fixed $s$, we show in Appendix B that the modular crossing equations labeled by $r$ with $\operatorname{gcd}(r, s)=1$ are solved by the term $\rho_{j, s}(\bar{h}+j, \bar{h})$ in the sum in (4.11). In particular, we show that, to leading order in $\beta \rightarrow 0$, the terms with $s^{\prime} \neq s$ do not contribute to the modular crossing equations for any $r$ at a fixed $s$.

Just as in Sec. III, where the density of states for the extended Cardy formula can be interpreted as a product of modular kernels for the $S$ transformation, the density of states we derived in this section can also be interpreted as modular kernels for more general $\operatorname{PSL}(2, \mathbb{Z})$ transformations. In Appendix D, we present simplified expressions for some of these modular kernels.

\section{TWIST GAP REVISITED}

Let us examine the solution (4.11) to the crossing equation. In the large spin limit $j \gg c$, the leading terms are

$$
\begin{aligned}
\rho_{j}^{0}(\bar{h})= & \rho_{j, 1}(h, \bar{h})+\rho_{j, 2}(h, \bar{h})+\mathcal{O}\left(e^{\frac{4 \pi}{3} \sqrt{\left(\frac{c-1}{24}\right) j}}\right) \\
= & {\left[d_{0}(h, 1)-d_{1}(h, 1)\right]\left[d_{0}(\bar{h}, 1)-d_{1}(\bar{h}, 1)\right] } \\
& +(-1)^{j}\left[d_{0}(h, 2)+d_{1}(h, 2)\right]\left[d_{0}(\bar{h}, 2)+d_{1}(\bar{h}, 2)\right] \\
& +\mathcal{O}\left(e^{\frac{4 \pi}{3} \sqrt{\left(\frac{c-1}{24}\right) j}}\right),
\end{aligned}
$$

where we write $\bar{h}+j=h$ above to avoid cluttering. Now we further focus on the following part of the spectrum in the large spin limit $j \gg c$ :

$0<\bar{h}-\frac{c-1}{24}<\frac{1}{8 \pi^{2}} \exp \left(-2 \pi \sqrt{j\left(\frac{c-1}{24}\right)}\right), \quad j:$ odd

In this regime, the first term $\rho_{j, 1}$ is smaller in magnitude than the second one $\rho_{j, 2}$. This is due to the different $\bar{h}$ dependence in $\rho_{j, 1}$ and $\rho_{j, 2}$. As $\bar{h}$ approaches $\frac{c-1}{24}$ from above, $\rho_{j, 1}$ scales as $\sqrt{\bar{h}-\frac{c-1}{24}}$, whereas $\rho_{j, 2}$ scales as $\frac{1}{\sqrt{\bar{h}-\frac{c-1}{24}}}$. In the region (5.2), the different $\bar{h}$ dependence is 
enough to overcome the larger exponential in spin that $\rho_{j, 1}$ has over $\rho_{j, 2}$. Moreover, $\rho_{j, s}$ for $s \geq 2$ scales as $\frac{1}{\sqrt{\bar{h}-\frac{c-1}{24}}}$ in the same regime, so we do not get qualitatively new behavior by considering higher $s$.

Since we take $j$ to be an odd integer, our crossing solution $\rho_{j}^{0}(\bar{h})$ is large and negative from the sign $(-1)^{j}$. In particular, in the large $j$ limit,

$$
\int_{\frac{c-1}{24}}^{\frac{c-1}{24}+\frac{1}{8 \pi^{2}} e^{-2 \pi \sqrt{j\left(\frac{c-1}{24}\right)}}} d \bar{h} \rho_{j}^{0}(\bar{h}) \simeq-\frac{\sqrt{2} e^{\pi \sqrt{j}}}{3 \pi \sqrt{j}} .
$$

The density of states of a physical CFT receives correction to the solution $\rho_{j}^{0}(\bar{h})$ from various sources. One obvious correction comes from the lowest twist $2 t_{\text {gap }}$ operator in the dual channel. Let the conformal weights of this lowest twist operator be $\left(t_{\text {gap }}, \bar{h}_{\text {gap }}\right) .{ }^{9}$ Repeating the same argument in Sec. III, this lowest twist operator contributes to the density of states by

$$
\begin{aligned}
\rho_{j}^{\text {non }-\mathrm{vac}}(\bar{h})= & \frac{2}{\sqrt{\left(h-\frac{c-1}{24}\right)\left(\bar{h}-\frac{c-1}{24}\right)}} \\
& \times \cosh \left[4 \pi \sqrt{\left(\frac{c-1}{24}-t_{\text {gap }}\right)\left(h-\frac{c-1}{24}\right)}\right] \\
& \times \cosh \left[4 \pi \sqrt{\left(\frac{c-1}{24}-\bar{h}_{\text {gap }}\right)\left(\bar{h}-\frac{c-1}{24}\right)}\right]
\end{aligned}
$$

in the large $j$ limit. Note that this contribution to the density of states is exponentially large in the large spin limit only if the twist $2 t_{\text {gap }}$ is below $\frac{c-1}{12}$, which is necessarily the case as we reviewed in Sec. II. This positive contribution from the lowest twist nonvacuum primary can only overcome the negativity of $\rho_{j}^{0}(\bar{h})$ in the regime (5.2) if

$$
t_{\text {gap }} \leq \frac{c-1}{32} \text {. }
$$

This leads to a tempting conclusion that all twodimensional, unitary, $c>1$ CFTs with unique normalizable vacua have twist gap $2 t_{\text {gap }}$ no greater than $\frac{c-1}{16} \cdot{ }^{10}$

\footnotetext{
${ }^{9}$ Here we implicitly assume there are only a finite number of low twist operators. More precisely, we assume there exists an $\epsilon>0$ such that there are a finite number of primary operators with $h<t_{\text {gap }}+\epsilon$. This is not necessarily the case: there could be an accumulation point in the twist. In Appendix $\mathrm{C}$ we will consider a slight generalization of the argument in this section where we account for this possibility, which suggests a weaker bound that $t_{\text {gap }} \leq \frac{15}{16}\left(\frac{c-1}{24}\right)$.

${ }^{10}$ In [22], a spin-dependent shift in the BTZ threshold is discussed, which can potentially be another way to cure the negative density of states.
}

This is not yet a rigorous derivation as there are potentially other corrections to the density of states that might cure the negativity in $\rho_{j}^{0}(\bar{h})$. Among other things, there are error terms from approximating the discrete spectrum by a continuous density of states as mentioned in Sec. III. It would be interesting to extend the analysis in [19-21] to control the error in a rigorous way. For a CFT with a finite twist gap (and hence necessarily irrational), there is generally no huge degeneracy at a given energy level, so we expect the error due to granularity to be much smaller than that of a rational CFT.

An equivalent, but perhaps more intuitive, explanation of our argument is the following. Let us define

$$
\rho_{j}^{\text {odd }}(\bar{h})=\frac{1-(-1)^{j}}{2} \rho_{j}(\bar{h}),
$$

i.e., the density of Virasoro primaries of odd spin. We can obtain a crossing equation for $\rho_{j}^{\text {odd }}(\bar{h})$ by combining (4.6) for $\frac{r}{s}=0$ and $\frac{r}{s}=\frac{1}{2}$ :

$$
\begin{aligned}
\sum_{j=0}^{\infty} & e^{-\beta j} \int_{t_{\text {gap }}}^{\infty} d \bar{h} \rho_{j}^{\text {odd }}(\bar{h}) e^{-\bar{\beta}\left(\bar{h}-\frac{c-1}{24}\right)} \\
= & \frac{\pi}{\sqrt{\beta \bar{\beta}}}\left(e^{\frac{4 \pi^{2} c-1}{\beta}{ }^{24}} e^{\frac{4 \pi^{2} c-1}{\beta} 24}\left(1-e^{-\frac{4 \pi^{2}}{\beta}}\right)\left(1-e^{-\frac{4 \pi^{2}}{\beta}}\right)\right. \\
& \left.-\frac{1}{2} e^{\frac{\pi^{2} c-1}{\beta} 24} e^{\frac{\pi^{2} c-1}{\beta} 24}\left(1+e^{-\frac{\pi^{2}}{\beta}}\right)\left(1+e^{-\frac{\pi^{2}}{\beta}}\right)\right) \\
& +\mathcal{O}\left(e^{\frac{4 \pi^{2}}{\beta}\left(\frac{c-1}{24}-t_{\text {gap }}\right)}\right)
\end{aligned}
$$

If we take the inverse Laplace transform of the RHS of (5.7), we would obtain the first two terms of (4.11); a twist gap of $\frac{c-1}{16}$ or below would cure the negativity in this density. ${ }^{11}$

In [1], the authors found a bootstrap upper bound on the twist gap $2 t_{\text {gap }}$ that is numerically close to the analytic bound $\frac{c-1}{12}$ reviewed in Sec. II. A simple partition function that saturates this bound comes from the $c \geq 1$ Liouville theory

$$
Z_{\text {Liouville }}(q, \bar{q}) \propto \frac{1}{\tau_{2}^{\frac{1}{2}}|\eta(q)|^{2}}
$$

Alternatively, we could consider the $c=1$ compact boson at any finite radius, but now viewed as a partition function for a $c>1$ theory by shifting the vacuum Casimir energy. In this new interpretation, there is no vacuum, while the original vacuum of the $c=1$ compact boson is now an

\footnotetext{
${ }^{11}$ Readers may notice that the RHS of (5.7) without the error term can become negative for $\bar{\beta}$ of $\mathcal{O}\left(e^{\frac{3 \pi^{2}}{\beta}}\right)$. However, our lightcone bootstrap analysis requires taking $\beta \rightarrow 0$ first. Thus this apparent negativity in the canonical ensemble cannot be trusted.
} 
$h=\bar{h}=\frac{c-1}{24}$ primary. A notable difference of this example from the Liouville case is that the spectrum of primaries is discrete and includes all spins.

At first sight, this seems to imply that one cannot lower the twist gap below $\frac{c-1}{12}$, which is in tension with our suggested twist gap $\frac{c-1}{16}$. However, recall that in our argument, it is crucial that there is a normalizable vacuum with $h=\bar{h}=0$ in the spectrum for us to perform the light-cone bootstrap. Neither the Liouville partition function nor the shifted compact boson partition function contains a vacuum state; therefore they need not obey the constraints we derived. Similarly in the numerical modular bootstrap analysis, it is difficult to impose the condition that there is a normalizable vacuum in the spectrum. We therefore predict that the functionals found in [1] would have zeros above the twist gap that "coalesce." In other words, as the truncation order in the derivative increases, the zeros become denser and denser rather than approach a fixed spectrum.

Interestingly, unlike in [23], the limit as the truncation order goes to infinity does not produce a nontrivial "extremal functional." In the previous paragraph, we argued that the limit would produce a functional that vanishes for all integer spins with twist at least $\frac{c-1}{12}$. However, in Sec. VI, we will review a construction by $[12,13]$ that inputs any single state with twist below $\frac{c-1}{12}$ and produces a modular invariant function by adding states all with twist at least $\frac{c-1}{12}$. Since the proposed extremal functional would vanish on the crossing equation for this modular invariant function, it must in addition vanish on all states with twist below $\frac{c-1}{12}$. Therefore the extremal functional approaches zero as the truncation order goes to infinity.

The twist $2 t=2 \min (h, \bar{h})=\frac{c-1}{16}$ has also appeared in other contexts. In [7], the authors introduced the notion of the Virasoro mean field theory, defined as the inversion of the vacuum Virasoro block for the sphere four-point function. While the Virasoro mean field theory by itself does not give a consistent four-point function of a physical theory, it approximates the large spin CFT data of any compact, unitary 2D CFT with nonzero twist gap. For identical external operators with conformal weight $(h, \bar{h})$, the authors show that the associated Virasoro mean field theory spectrum is qualitatively different for $h>\frac{c-1}{32}$ versus $h<\frac{c-1}{32}$ : In the former case, the spectrum consists only of a continuum above $h>\frac{c-1}{24}$, while in the latter case, there are in addition a discrete set of primaries. ${ }^{12}$ Indeed, the fourpoint function of identical scalar primaries with scaling dimension $\Delta=\frac{c-1}{16}$ is special. The four-point sphere

\footnotetext{
${ }^{12}$ Note that in terms of the Liouville momentum $\alpha(h)>0$, defined as $h=\alpha(Q-\alpha)$ with $c=1+6 Q^{2}$, we have $2 \alpha\left(\frac{c-1}{32}\right)=\alpha\left(\frac{c-1}{24}\right)$.
}

conformal block with external scalar scaling dimension $\frac{c-1}{16}$ and internal scalar scaling dimension $\frac{c-1}{12}$ is a simple power of $|z|,|1-z|$, and is self-crossing invariant by itself [see, e.g., (3.15) of [24] ]. Furthermore, it has been shown in [25] that $\frac{c-1}{16}$ is the minimal external scalar scaling dimension for a four-point function with only internal scalar primaries. ${ }^{13}$ This value of the twist has also appeared in the discussion of the Renyi entropy after a local quench $[6,8]$.

\section{PURE GRAVITY}

The twist gap that we have proposed has very interesting implications for two-dimensional CFTs holographically dual to large-radius Einstein gravity in $\mathrm{AdS}_{3}$. Recall that the classical BTZ black hole has mass, $M$, and angular momentum, $j$, related to the CFT conformal dimensions $h$ and $\bar{h}$ via [26]

$$
M=\frac{1}{\ell_{\mathrm{AdS}}}\left(h+\bar{h}-\frac{c}{12}\right), \quad j=h-\bar{h}
$$

in the large $c$ limit. In particular, classical BTZ black holes have $M \ell_{\text {AdS }} \geq|j|$, which implies $h, \bar{h} \geq \frac{c}{24}+\mathcal{O}(1)$.

There have been attempts to formulate a "pure" theory of quantum gravity in $\mathrm{AdS}_{3}$ [12,13]. Via AdS/CFT, the strictest definition of a pure theory of gravity is a $2 \mathrm{D}$ unitary CFT at large $c$ where all nonvacuum Virasoro primary operators can be interpreted as BTZ black holes. ${ }^{14}$ The bound (5.5) on the twist gap suggested by our argument would imply that no such CFT exists. In this section we check that the pure gravity partition function computed in $[12,13]$ indeed has a negative density of states in the regime (5.2), confirming our general argument.

Our main result (4.11) is the universal contribution from the vacuum character in the dual channel to the large spin density of states. Its expression is very reminiscent of the sum over geometries in the calculation of the partition function of pure $\mathrm{AdS}_{3}$ gravity [12,13]. This is not a coincidence. We will further show that in the limit $\bar{h}-\frac{c-1}{24} \rightarrow 0$ and $j \gg c$, the pure gravity partition function matches our $\rho_{j}^{0}(\bar{h})$ (4.11). We therefore reach an important

\footnotetext{
${ }^{13}$ If the external scaling dimension is greater than or equal to $\frac{c-1}{12}$, then such four-point functions with only scalar internal primaries are realized by Liouville theory. If the external scaling dimension is between $\frac{c-1}{16}$ and $\frac{c-1}{12}$, such four-point functions can be obtained by analytically continuing the Liouville four-point function [25].

${ }^{14}$ The extremal partition functions of [27] are also often referred to as partition functions of pure gravity. However, due to their holomorphic factorization, the partition functions of [27] contain nonvacuum states with a vanishing twist that therefore cannot be interpreted as BTZ black holes. In this section we thus will focus on the partition function of $[12,13]$, which obeys the stricter definition of pure gravity.
} 
conclusion: Even though the pure gravity partition function derived in $[12,13]$ has various unphysical properties, it is the universal contribution from the vacuum character to the density of large spin states in any CFT with a finite twist gap.

\section{A. Maloney-Witten-Keller partition function}

The Maloney-Witten-Keller (MWK) partition function $[12,13]$ is computed by starting with the vacuum Virasoro character

$$
\begin{aligned}
\chi_{0}(q) \chi_{0}(\bar{q})= & \frac{1}{|\eta(q)|^{2}}\left(q^{-\frac{c-1}{24}} \bar{q}^{-\frac{c-1}{24}}-q^{-\frac{c-1}{24}+1} \bar{q}^{-\frac{c-1}{24}}\right. \\
& \left.-q^{-\frac{c-1}{24}} \bar{q}^{-\frac{c-1}{24}+1}+q^{-\frac{c-1}{24}+1} \bar{q}^{-\frac{c-1}{24}+1}\right),
\end{aligned}
$$

and adding its $\operatorname{PSL}(2, \mathbb{Z})$ images. The sum is divergent, and a certain regularization is required to make the answer finite. The MWK partition function has the following features:

(i) It has a unique vacuum, and all other Virasoro primaries have $h, \bar{h} \geq \frac{c-1}{24}$.

(ii) The spectrum contains a continuum of states with integer spins.

(iii) The density of states is not always positive. In particular, the degeneracy of the state with $h=\bar{h}=$ $\frac{c-1}{24}$ is -6 .

Since the MWK partition function has no nonvacuum state with twist below $\frac{c-1}{16}$, our argument in Sec. V suggests that the density of states must turn negative in the regime (5.2), in addition to the known negativity at $h=\bar{h}=\frac{c-1}{24}$. We will show that this is exactly the case.

Below we review the density of states for the MWK partition function. Instead of using the spin $j$ and the twist $2 t$, we will follow the convention in [13] and use the variables $e$ and $j$ defined as

$$
e=h+\bar{h}-\frac{c-1}{12}, \quad j=h-\bar{h} .
$$

The density of states for the MWK partition function receives contributions from the $\operatorname{PSL}(2, \mathbb{Z})$ sums from each of the four terms $\frac{1}{|\eta(q)|^{2}} q^{E_{L}} \bar{q}^{E_{R}}$ in (6.2),

$\rho_{j}^{\mathrm{MWK}}(e)=\rho_{j}^{-\frac{c-1}{12}, 0}(e)-\rho_{j}^{-\frac{c-13}{12}, 1}(e)-\rho_{j}^{-\frac{c-13}{12},-1}(e)+\rho_{j}^{-\frac{c-25}{12}, 0}(e)$,

where the superscripts $E, J$ of $\rho_{j}^{E, J}(e)$ are defined analogously as $E=E_{L}+E_{R}, J=E_{L}-E_{R}$ for each of the "seed" terms $\frac{1}{|\eta(q)|^{2}} q^{E_{L}} \bar{q}^{E_{R}}$ in (6.2).

Each of the four terms in (6.4) is further written as an infinite sum:

$$
\rho_{j}^{E, J}(e)=\sum_{m=0}^{\infty} \rho_{j, m}^{E, J}(e)
$$

When $j \neq 0$, which is the case of interest, $\rho_{j, m}^{E, J}(e)$ is given by

$\rho_{j, m}^{E, J}(e)=Z_{j, J}(m+1 / 2) \frac{2^{3 m+1} \pi^{2 m}}{(2 m) !}|j|^{m-1} \nu_{m}^{E, J}(e /|j|)$,

where the function $\nu_{m}^{E, J}(t)$ will be defined momentarily. ${ }^{15}$ $Z_{j, J}(m+1 / 2)$ is the Kloosterman zeta function defined by a Dirichlet series

$$
Z_{j, J}(m+1 / 2)=\sum_{s=1}^{\infty} s^{-2(m+1 / 2)} S(j, J ; s) .
$$

When $m=0$, the above series diverges and the Kloosterman zeta function $Z_{j, J}(1 / 2)$ is defined by analytic continuation and finite of order $\mathcal{O}\left(j^{2} J^{2}\right)$ [12,13]. For our purposes, the dominant contributions come from the higher $m$ terms, so we will not be concerned about the explicit regularized values of $Z_{j, J}(1 / 2)$.

Below we define the function $\nu_{m}^{E, J}(t)$. Since all of the nonvacuum states in the MWK partition function have $h, \bar{h} \geq \frac{c-1}{24}$, i.e., $e \geq|j|$, we will define the function $\nu_{m}^{E, J}(t)$ only for $t \geq 1$. We first define

$$
f_{m}(t)=\frac{1}{\sqrt{t^{2}-1}} \cosh \left(m \cosh ^{-1}(t)\right) .
$$

Next, we define the operator $D_{t}$ as

$D_{t} f_{k}=-E f_{k}-J f_{k-1}-\frac{J}{2}\left(1-\frac{m}{k}\right)\left(f_{k+1}-f_{k-1}\right)$,

which can be realized by a matrix action. With these preparations, $\nu_{m}^{E, J}(t)$ is given by

$$
\nu_{m}^{E, J}(t)=D_{t}^{m} f_{m}(t)
$$

\section{B. Double limit}

Here we consider a special case of the double limit (5.2) where we first take $\bar{h}$ to $\frac{c-1}{24}$, and then take $j$ to be odd and large. The function $\nu_{m}^{E, J}(t)$ simplifies in the limit $t=e /|j| \rightarrow 1$ :

$$
\nu_{m}^{E, J}(t) \simeq \sqrt{\frac{1}{2(t-1)}}(-E-J)^{m} .
$$

\footnotetext{
${ }^{15} \nu_{m}^{E, J}(e /|j|)$ is denoted as $\nu_{j, m}(e)$ in [13].
} 
Hence in this limit, we have ${ }^{16}$

$$
\begin{aligned}
\rho_{j}^{E, J}(e) \simeq & \sum_{s=1}^{\infty} \frac{2}{s} S(j, J ; s) \\
& \sum_{m=1}^{\infty} s^{-2 m} \frac{2^{3 m} \pi^{2 m}}{(2 m) !}|j|^{m-1}(-E-J)^{m} \sqrt{\frac{j}{4\left(\bar{h}-\frac{c-1}{24}\right)}} .
\end{aligned}
$$

In the large spin $j$ limit, the sum is dominated by large $m$, and we can approximate the sum in $m$ by an exponential:

$$
\begin{aligned}
\rho_{j}^{E, J}(e) \simeq & \sum_{s=1}^{\infty} \frac{1}{2 s \sqrt{j\left(\bar{h}-\frac{c-1}{24}\right)}} S(j, J ; s) \\
& \times \exp \left(\frac{4 \pi}{s} \sqrt{\frac{-E-J}{2} j}\right) .
\end{aligned}
$$

Adding up the four terms in (6.4), we obtain the following expression for the MWK density of states in the limit where we take $\bar{h} \rightarrow \frac{c-1}{24}$ first and then $j \rightarrow \infty$ :

$$
\begin{aligned}
\rho_{j}(e) \simeq & \sum_{s=1}^{\infty} \frac{1}{2 s \sqrt{j\left(\bar{h}-\frac{c-1}{24}\right)}}\left[S(j, 0 ; s) \exp \left(\frac{4 \pi}{s} \sqrt{\frac{c-1}{24} j}\right)\right. \\
& +S(j, 0 ; s) \exp \left(\frac{4 \pi}{s} \sqrt{\frac{c-25}{24} j}\right) \\
& -S(j,-1 ; s) \exp \left(\frac{4 \pi}{s} \sqrt{\frac{c-1}{24} j}\right) \\
& \left.-S(j, 1 ; s) \exp \left(\frac{4 \pi}{s} \sqrt{\frac{c-25}{24}} j\right)\right]
\end{aligned}
$$

which is precisely our formula (4.11) for the asymptotic density of states in this limit. ${ }^{17}$ In particular, the $s=1$ term in (6.14) vanishes and the density of states is dominated by the $s=2$ term, which is negative in this limit as argued in Sec. V. Furthermore, we can show that the MWK density of states is negative in the more general regime (5.2) as predicted by (4.11) by keeping the subleading term of $\nu_{m}^{E, J}(t)$ in the $t \rightarrow 1$ limit, namely

$$
\nu_{m}^{E, J}(t)=\sqrt{\frac{1}{2(t-1)}}(-E-J)^{m}+\frac{(2 m-1)(-E-J)^{m-1}((2 m+1)(-E)+(2 m-1) J) \sqrt{t-1}}{4 \sqrt{2}}+\mathcal{O}\left((t-1)^{\frac{3}{2}}\right) .
$$

This gives the subleading term in $\left(\bar{h}-\frac{c-1}{24}\right)$ when plugged into $\rho_{j}(e)$. In particular, this accounts for the leading nonzero contribution at $s=1$. We have also confirmed our prediction (4.11) numerically with the MWK density of states.

In [13], the authors show that the density of states is positive if we fix $e$ and $j$, and then take $c$ to be large. Here we uncover the negative density of states in a different regime (5.2) where both $e$ and $j$ are taken to be much larger than $c$. This new regime of negative density of states makes it more challenging to correct the MWK partition function to a unitary, physical partition function.

To cancel the negative density of states in the regime (5.2) of the MWK partition function without ruining modular invariance, one tentative candidate is to add $N$ copies of the $\operatorname{PSL}(2, \mathbb{Z})$ sum of the state $(h, \bar{h})=\left(\frac{c-1}{32}, \frac{c-1}{32}\right)$ above the vacuum. The "seed" term of this addition to the

\footnotetext{
${ }^{16}$ Strictly speaking, the $m=0$ term requires analytic continuation to make sense, but in the following we will only be interested in the large $m$ terms which dominate in the limit (5.2).

${ }^{17}$ In comparing the above with (4.11), there is a relative factor of 2 coming from the Jacobian factor when we change variables from $t, j$ to $e, j$.
}

partition function is $\frac{N}{|\eta(q)|^{2}} q^{\frac{c-1}{32}-\frac{c-1}{24}} \bar{q}^{\frac{c-1}{32}-\frac{c-1}{24}}=\frac{N}{|\eta(q)|^{2}} q^{-\frac{c-1}{96}} \bar{q}^{-\frac{c-1}{96}}$, and therefore contributes to the density of states by $N \rho^{E=-\frac{c-1}{48}, J=0}$. As shown in [13], $\rho_{j}^{E, 0}(e)$ (with $E<0$ ) is positive everywhere except for a negative delta function at $e=0$ with $j=0$. This delta function negativity can be canceled by adding, for example, the modular invariant partition function of the $c=1$ self-dual boson (see Sec. 4.2 of [13]). Putting everything together, let us consider the final density of states

$$
\rho_{j}^{\mathrm{MWK}}(e)+N \rho^{-\frac{c-1}{48}, 0}(e)+(N+6) \rho_{j}^{c=1}(e),
$$

where $\rho_{j}^{c=1}(e)$ is the density of states for the $c=1$ self-dual boson, whose vacuum is now interpreted as a $h=\bar{h}=\frac{c-1}{24}$ state. The term $6 \rho_{j}^{c=1}(e)$ is there to cancel the negative delta function density of states at $h=\bar{h}=\frac{c-1}{24}$ coming from the MWK partition function itself. For $N>1$, the above density of states appears to be positive at large $c$ in all regimes we have considered, and gives a modular invariant partition function. It has a vacuum, a finite number of states at $h=\bar{h}=\frac{c-1}{32}$, and a continuum starting at $h, \bar{h} \geq \frac{c-1}{24}$. The twist gap $2 t_{\text {gap }}$ and the gap in the scaling dimension $\Delta_{\text {gap }}$ 
are both $\frac{c-1}{16}$. It would be interesting to prove that this density of states is indeed positive everywhere. ${ }^{18}$

\section{SUPERSYMMETRIC GENERALIZATION}

Our arguments in this paper can be generalized to 2D CFTs with any chiral algebra. In this section we will perform a similar analysis for the $\mathcal{N}=1$ super-Virasoro algebra. Recall that there are four partition functions we can consider, depending on the four spin structures on the torus, which correspond to (anti)periodic boundary conditions of the fermions in the space and time directions. ${ }^{19}$ These correspond to partition functions restricted to the NeveuSchwarz or Ramond sectors, and with or without a $(-1)^{F}$ insertion. Three of these partition functions are related by $\operatorname{PSL}(2, \mathbb{Z})$ transformations, and the remaining one is the Witten index.

In this section we will focus on the partition function with antiperiodic boundary conditions for the fermions in both directions on the torus, namely

$$
Z(q, \bar{q})=\operatorname{Tr}_{\mathcal{H}_{\mathrm{NS}, \mathrm{NS}}}\left(q^{L_{0}-\frac{c}{24}} \bar{q}^{\bar{L}_{0}-\frac{c}{24}}\right) .
$$

This function is invariant under a subgroup of $\operatorname{PSL}(2, \mathbb{Z})$, generated by the group elements $S$ and $T^{2}$. We will denote this group as $\Gamma_{\theta}$, which can also be defined by $\operatorname{SL}(2, \mathbb{Z})$ matrices $\left(\begin{array}{ll}a & b \\ c & d\end{array}\right)$ with $a+d$ and $b+c$ both even. The characters for the $\mathcal{N}=1$ super-Virasoro algebra with $c>\frac{3}{2}$ under this spin structure are

$$
\begin{aligned}
& \chi_{0}^{\mathcal{N}=1}(q)=q^{-\frac{c-\frac{3}{2}}{24}}\left(1-q^{\frac{1}{2}}\right) \frac{\eta(q)}{\eta\left(q^{2}\right) \eta\left(q^{1 / 2}\right)} \quad \text { vacuum, } \\
& \chi_{h}^{\mathcal{N}=1}(q)=q^{h-\frac{c-\frac{3}{2}}{24}} \frac{\eta(q)}{\eta\left(q^{2}\right) \eta\left(q^{1 / 2}\right)}, \quad h>0 .
\end{aligned}
$$

We can similarly define a modular kernel $K_{S}^{\mathcal{N}=1}(h)$ as

$$
\chi_{0}^{\mathcal{N}=1}\left(q^{\prime}\right)=\int_{0}^{\infty} d h K_{S}^{\mathcal{N}=1}(h) \chi_{h}^{\mathcal{N}=1}(q)
$$

where $q^{\prime}$ is the $S$ transform of $q$. It is given by

\footnotetext{
${ }^{18}$ Note that there is a simple alternative to (6.16) where instead of adding scalars of twist $\frac{c-1}{16}$ to the MWK partition function, we add twist $\frac{c-1}{16}$ states with arbitrary spin [plus $\operatorname{PSL}(2, \mathbb{Z})$ images]. If the spectra of these partition functions are also positive definite, they would have twist gap $\frac{c-1}{16}$ and scaling gap no greater than $\frac{c-1}{12}$.

${ }^{19}$ Here we assume for simplicity that left and right moving fermions have identical spin structures. It would be interesting to explore modular constraints by considering partition functions with mixed spin structures.
}

$$
\begin{aligned}
K_{S}^{\mathcal{N}=1}(h) & =\sqrt{\frac{2}{h-\frac{c-\frac{3}{2}}{24}}} \theta\left(h-\frac{c-\frac{3}{2}}{24}\right) \\
& \times\left[\cosh \left(4 \pi \sqrt{\left(\frac{c-\frac{3}{2}}{24}\right)\left(h-\frac{c-\frac{3}{2}}{24}\right)}\right)\right. \\
& \left.-\cosh \left(4 \pi \sqrt{\left(\frac{c-\frac{27}{2}}{24}\right)\left(h-\frac{c-\frac{3}{2}}{24}\right)}\right)\right] .
\end{aligned}
$$

By the same argument in Sec. III, any 2D CFT with $\mathcal{N}=1$ super-Virasoro symmetry and a nonzero twist gap under the $\mathcal{N}=1$ super-Virasoro algebra obeys a Cardylike formula with extended validity. In particular, the density of super-Virasoro primaries is given by

$$
\rho_{j, 1}^{0}(\bar{h})=K_{S}^{\mathcal{N}=1}(\bar{h}+j) K_{S}^{\mathcal{N}=1}(\bar{h}),
$$

which is valid whenever $\bar{h}+j \gg c, \bar{h}>\frac{c-\frac{3}{2}}{24}$.

However, just as for the nonsupersymmetric case, Eq. (7.5) is not all we can learn from the light-cone modular bootstrap. Let us consider a more general setup where we set $\tau$ and $\bar{\tau}$ as in (4.1), in the limit where $\beta$ goes to zero with $\bar{\beta}$ fixed. Again, $r$ and $s$ in (4.1) are coprime integers, and $s \geq 1$; we will now in addition demand that $r$ and $s$ have different parities. We can consider a $\Gamma_{\theta}$ transformation

$$
\left(\begin{array}{cc}
a(r, s) & b(r, s) \\
s & -r
\end{array}\right) \text {. }
$$

For consistency, $a(r, s)$ must satisfy the following properties: If $s$ is even, $a(r, s)=-\left(r^{-1}\right)_{2 s}$. If $s$ is odd, $a(r, s)=-\left(r^{-1}\right)_{s}$ and chosen so that $a(r, s)$ is even. We then obtain the following crossing equation [similar to (4.6) for the Virasoro case]:

$$
\begin{aligned}
& \sum_{\substack{j>0 \\
j \in \bar{Z} / 2}} \exp \left[-\left(\beta-\frac{2 \pi i r}{s}\right) j\right] \int d \bar{h} \rho_{j}(\bar{h}) \exp \left[-\bar{\beta}\left(\bar{h}-\frac{c-\frac{3}{2}}{24}\right)\right] \\
& =\frac{2 \pi}{s \sqrt{\beta \bar{\beta}}} e^{\frac{4 \pi^{2}\left(c-\frac{3}{2}\right)}{24 s^{2} \beta}} e^{\frac{4 \pi^{2}\left(c-\frac{3}{2}\right)}{24 s^{2} \bar{\beta}}}\left(1-e^{-\frac{2 \pi^{2}}{s^{2} \beta}+\frac{\pi i a(r, s)}{s}}\right)\left(1-e^{-\frac{2 \pi^{2}}{s^{2} \bar{\beta}}-\frac{\pi i a(r, s)}{s}}\right) \\
& +\mathcal{O}\left(e^{\frac{4 \pi^{2}}{s^{2} \beta}\left(\frac{c-\frac{3}{2}}{24}-t_{\text {gap }}\right)}\right) .
\end{aligned}
$$

In (7.7), the sum over $j$ runs over both integers and half-integers.

We will find a continuous solution to (7.7), in a similar manner as in Sec. IV B. We define a $\Gamma_{\theta}$ Kloosterman sum as

$$
S^{\Gamma_{\theta}}(j, J ; s)=\sum_{\substack{r: \text { gcd }(r, s)=1 \\ 0 \leq r<2 s \\ r+s \text { odd }}} \exp \left(\pi i \frac{2 r j-a(r, s) J}{s}\right),
$$

which satisfies

$$
\sum_{\substack{j=0 \\ j \in \mathbb{Z} / 2}}^{\operatorname{lcm}\left(s, s^{\prime}\right)-\frac{1}{2}} S^{\Gamma_{\theta}}\left(j, J, s^{\prime}\right) e^{-\frac{2 \pi i r j}{s}}=2 s e^{-\frac{\pi i a(r, s) J}{s}} \delta_{s, s^{\prime}} .
$$


We also define the functions

$$
\begin{aligned}
& d_{0}^{\mathcal{N}=1}(h, s)=\sqrt{\frac{2}{s\left(h-\frac{c-\frac{3}{2}}{24}\right)}} \cosh \left(\frac{4 \pi}{s} \sqrt{\left(h-\frac{c-\frac{3}{2}}{24}\right)\left(\frac{c-\frac{3}{2}}{24}\right)}\right) \theta\left(h-\frac{c-\frac{3}{2}}{24}\right), \\
& d_{1}^{\mathcal{N}=1}(h, s)=\sqrt{\frac{2}{s\left(h-\frac{c-\frac{3}{2}}{24}\right)}} \cosh \left(\frac{4 \pi}{s} \sqrt{\left.\left(h-\frac{c-\frac{3}{2}}{24}\right)\left(\frac{c-\frac{27}{2}}{24}\right)\right) \theta\left(h-\frac{c-\frac{3}{2}}{24}\right),},\right.
\end{aligned}
$$

and

$$
\begin{aligned}
\rho_{j, s}^{\mathcal{N}=1}(h, \bar{h})= & S^{\Gamma_{\theta}}(j, 0 ; s) d_{0}^{\mathcal{N}=1}(h, s) d_{0}^{\mathcal{N}=1}(\bar{h}, s) \\
& -S^{\Gamma_{\theta}}(j,-1 ; s) d_{0}^{\mathcal{N}=1}(h, s) d_{1}^{\mathcal{N}=1}(\bar{h}, s) \\
& -S^{\Gamma_{\theta}}(j, 1 ; s) d_{1}^{\mathcal{N}=1}(h, s) d_{0}^{\mathcal{N}=1}(\bar{h}, s) \\
& +S^{\Gamma_{\theta}}(j, 0 ; s) d_{1}^{\mathcal{N}=1}(h, s) d_{1}^{\mathcal{N}=1}(\bar{h}, s) .
\end{aligned}
$$

A solution to (7.7) at large spin is then given by

$$
\rho_{j}^{0}(\bar{h})=\sum_{s^{\prime}=1} \rho_{j, s^{\prime}}^{\mathcal{N}=1}(\bar{h}+j, \bar{h}), \quad j \gg c .
$$

The solution (7.12) again has the interesting property that for large odd (or half-integer) spin, with $\bar{h}$ exponentially close to $\frac{c-\frac{3}{2}}{24}$, there is a negative degeneracy of states. This can be cured with a single operator whose twist is at or below $\frac{c-\frac{3}{2}}{16}$.

\section{ACKNOWLEDGMENTS}

We thank S. Collier, T. Hartman, S. Kachru, Z. Komargodski, Y.-H. Lin, R. Mahajan, A. Maloney, H. Maxfield, D. Mazac, B. Mukhametzhanov, E. Perlmutter, L. Rastelli, D. Simmons-Duffin, D. Stanford, $\mathrm{H}$. Verlinde, and E. Witten for interesting discussions. We thank T. Hartman, C. Keller, A. Maloney, H. Maxfield, and $\mathrm{X}$. Yin for comments on a draft. The work of N. B. is supported in part by the Simons Foundation Grant No. 488653. The work of H.O. is supported in part by U.S. Department of Energy Grant No. DE-SC0011632, by the World Premier International Research Center Initiative, MEXT, Japan, by JSPS Grant-in-Aid for Scientific Research C-26400240, and by JSPS Grant-in-Aid for Scientific Research on Innovative Areas 15H05895. The work of S. H.S. is supported by the National Science Foundation Grant No. PHY-1606531 and by the Roger Dashen Membership. The work of Y.W. is supported in part by the U.S. NSF under Grant No. PHY-1620059 and by the Simons Foundation Grant No. 488653. N. B. and H. O. thank the Aspen Center for Theoretical Physics, which is supported by the National Science Foundation Grant No. PHY-1607611, where part of this work was done.

\section{APPENDIX A: SUM ESTIMATION}

Consider a complex function $f(z)$ that satisfies the following properties:

(i) $f(z)$ is analytic on the half-plane $\operatorname{Re} z \geq 0$.

(ii) $\lim _{|\operatorname{Im} z| \rightarrow \infty} e^{-2 \pi|\operatorname{Im} z|}|f(z)|=0$ uniformly in any finite interval $(\delta, \Lambda)$ of $\operatorname{Re} z$.

From a contour argument, one can derive the Abel-Plana formula (see Sec. III in [28] or Sec. 13.14 in [29]) which relates the discrete sum to the integral of $f(z)$,

$$
\begin{aligned}
\sum_{k=0}^{\infty} & f(k)-\int_{0}^{\infty} d z f(z) \\
= & \frac{1}{2} f(0)+i \int_{0}^{\infty} d z \frac{f(i z)-f(-i z)}{e^{2 \pi z}-1} \\
& +\lim _{\Lambda \rightarrow+\infty}\left(\frac{1}{2} f(\Lambda)+i \int_{0}^{\infty} d z \frac{f(\Lambda-i z)-f(\Lambda+i z)}{e^{2 \pi z}-1}\right) .
\end{aligned}
$$

One application of the formula arises in the main text where we replace the sum over spin $j$ by an integral over $j$ in (3.7). In this case, we choose

$$
\begin{aligned}
f(j)= & e^{-\beta j} \int_{\frac{c-1}{24}}^{\infty} d \bar{h} \frac{e^{4 \pi \sqrt{(\bar{h}+j)\left(\frac{c-1}{24}\right)}} K_{S}(\bar{h})}{\sqrt{\bar{h}+j-\frac{c-1}{24}}} \\
& \times \exp \left[-\bar{\beta}\left(\bar{h}-\frac{c-1}{24}\right)\right],
\end{aligned}
$$

which clearly satisfies the criterion for the Abel-Plana formula and in addition $\lim _{\Lambda \rightarrow+\infty} f(\Lambda+i y)=0$. Moreover the RHS of (A1) is finite in the limit $\beta \rightarrow 0$; thus we can freely replace the sum with the integral (or vice versa) without affecting the exponentially dominating terms of the form $\mathcal{O}\left(e^{\frac{a}{\beta}}\right)$ for some $\beta$-independent constant $a$.

\section{APPENDIX B: CROSSING SOLUTION}

In this Appendix we show that (4.11) solves the crossing equation (4.6). In particular, we show that for the modular crossing equation (4.6) labeled by $s$ (and for all coprime $1 \leq r<s$ ), only the $\rho_{j, s^{\prime}=s}$ term in (4.11) contributes to the leading term on the RHS of (4.6) in the $\beta \rightarrow 0$ limit.

The LHS of (4.6) is given by (see end of Sec. IV B for the range of $s^{\prime}$ ) 


$$
\begin{aligned}
\sum_{j=0}^{\infty} e^{\frac{2 \pi i r j}{s}} \exp [-j \beta] \int_{t_{\mathrm{gap}}}^{\infty} d \bar{h} \rho_{j}(\bar{h}) \exp \left[-\bar{\beta}\left(\bar{h}-\frac{c-1}{24}\right)\right] \\
=\sum_{s^{\prime}} \sum_{j=0}^{\infty} e^{\frac{2 \pi i r j}{s}} \exp [-j \beta] \int_{t_{\mathrm{gap}}}^{\infty} d \bar{h} \exp \left[-\bar{\beta}\left(\bar{h}-\frac{c-1}{24}\right)\right] \\
\quad \times\left[S\left(j, 0 ; s^{\prime}\right) d_{0}\left(\bar{h}+j, s^{\prime}\right) d_{0}\left(\bar{h}, s^{\prime}\right)-S\left(j,-1 ; s^{\prime}\right) d_{0}\left(\bar{h}+j, s^{\prime}\right) d_{1}\left(\bar{h}, s^{\prime}\right)\right. \\
\left.\quad-S\left(j, 1 ; s^{\prime}\right) d_{1}\left(\bar{h}+j, s^{\prime}\right) d_{0}\left(\bar{h}, s^{\prime}\right)+S\left(j, 0 ; s^{\prime}\right) d_{1}\left(\bar{h}+j, s^{\prime}\right) d_{1}\left(\bar{h}, s^{\prime}\right)\right] .
\end{aligned}
$$

Let us consider the first term coming from the square bracket on the RHS of (B1). We rewrite $j=\tilde{j}+k \operatorname{lcm}\left(s, s^{\prime}\right)$ where $k \geq 0$ and $0 \leq \tilde{j} \leq \operatorname{lcm}\left(s, s^{\prime}\right)-1$ to get

$$
\begin{aligned}
\sum_{s^{\prime}} & \sum_{j=0}^{\infty} e^{\frac{2 \pi i r j}{s}} \exp [-j \beta] \int_{t_{\mathrm{gap}}}^{\infty} d \bar{h} S\left(j, 0 ; s^{\prime}\right) d_{0}\left(\bar{h}+j, s^{\prime}\right) d_{0}\left(\bar{h}, s^{\prime}\right) \exp \left[-\bar{\beta}\left(\bar{h}-\frac{c-1}{24}\right)\right] \\
= & \sum_{s^{\prime}} \sum_{\tilde{j}=0}^{\operatorname{lcm}\left(s, s^{\prime}\right)-1} e^{\frac{2 \pi i r \tilde{j}}{s}} S\left(\tilde{j}, 0 ; s^{\prime}\right) \sum_{k=0}^{\infty} \exp \left[-\left(\tilde{j}+k \operatorname{lcm}\left(s, s^{\prime}\right)\right) \beta\right] \\
& \times \int_{t_{\mathrm{gap}}}^{\infty} d \bar{h} d_{0}\left(\bar{h}+\tilde{j}+k \operatorname{ccm}\left(s, s^{\prime}\right), s^{\prime}\right) d_{0}\left(\bar{h}, s^{\prime}\right) \exp \left[-\bar{\beta}\left(\bar{h}-\frac{c-1}{24}\right)\right] .
\end{aligned}
$$

If we replaced the sum over $k$ with an integral in (B2), we could shift variables $k \rightarrow k-\frac{\tilde{j}}{\operatorname{lcm}\left(s, s^{\prime}\right)}$. In Appendix A, we show that the correction coming from changing the sum to an integral approaches a constant as $\beta$ goes to 0 . Since we only aim to reproduce the divergence as $\beta \rightarrow 0$ on the RHS of (4.6), we can approximate the sum in $k$ by an integral,

$\sum_{s^{\prime}}\left[\sum_{\tilde{j}=0}^{\operatorname{lcm}\left(s, s^{\prime}\right)-1} e^{2 \pi i r \tilde{j}} S\left(\tilde{j}, 0 ; s^{\prime}\right)\right] \int_{0}^{\infty} d k \int_{t_{\text {gap }}}^{\infty} d \bar{h} d_{0}\left(\bar{h}+k \operatorname{lcm}\left(s, s^{\prime}\right), s^{\prime}\right) d_{0}\left(\bar{h}, s^{\prime}\right) \exp \left[-\beta k \operatorname{lcm}\left(s, s^{\prime}\right)\right] \exp \left[-\bar{\beta}\left(\bar{h}-\frac{c-1}{24}\right)\right]$.

The sum over $\tilde{j}$ in (B3) can now be done using (4.8), which is proportional to $\delta_{s, s^{\prime}}$. It follows that only the $\rho_{j, s^{\prime}=s}$ term in the sum (4.11) contributes to the modular crossing equation (4.6) labeled by $s$. The $s^{\prime}=s$ term then gives

$$
\begin{aligned}
s & \int_{0}^{\infty} d k \int_{t_{\mathrm{gap}}}^{\infty} d \bar{h} d_{0}(\bar{h}+k s, s) d_{0}(\bar{h}, s) \exp \left[-\bar{\beta}\left(\bar{h}-\frac{c-1}{24}\right)-\beta k s\right] \\
& =\int_{t_{\mathrm{gap}}}^{\infty} d \bar{h} \exp [\beta \bar{h}] \int_{\bar{h}}^{\infty} d k d_{0}(k, s) d_{0}(\bar{h}, s) \exp \left[-\bar{\beta}\left(\bar{h}-\frac{c-1}{24}\right)-\beta k\right] \\
& =\sqrt{\frac{2 \pi}{s \beta}} e^{\frac{4 \pi^{2}(c-1)}{24 s^{2} \beta}} \int_{t_{\mathrm{gap}}}^{\infty} d \bar{h} \exp \left[(\beta-\bar{\beta})\left(\bar{h}-\frac{c-1}{24}\right)\right] d_{0}(\bar{h}, s) \\
& \simeq \sqrt{\frac{2 \pi}{s \beta}} e^{\frac{4 \pi^{2}(c-1)}{24 s^{2} \beta}} \int_{t_{\mathrm{gap}}}^{\infty} d \bar{h} \exp \left[-\bar{\beta}\left(\bar{h}-\frac{c-1}{24}\right)\right] d_{0}(\bar{h}, s) \\
& =\frac{2 \pi}{s \sqrt{\beta \bar{\beta}}} e^{\frac{4 \pi^{2}(c-1)}{24 s^{2} \beta}} e^{\frac{4 \pi^{2}(c-1)}{24 s^{2} \bar{\beta}}}
\end{aligned}
$$


where we use $\simeq$ to mean equal in the limit $\beta \rightarrow 0$. We have succeeded in reproducing one of the divergent terms on the RHS of (4.6).

We can evaluate the remaining three terms in (B1) following the same steps. When combined, we finally get

$\frac{2 \pi}{s \sqrt{\beta \bar{\beta}}} e^{\frac{4 \pi^{2}(c-1)}{24 s^{2} \beta}} e^{\frac{4 \pi^{2}(c-1)}{24 s^{2} \bar{\beta}}}\left(1-e^{-\frac{4 \pi^{2}}{s^{2}}-\frac{2 \pi i\left(r^{-1}\right) s}{s}}\right)\left(1-e^{-\frac{4 \pi^{2}}{s^{2} \bar{\beta}}+\frac{2 \pi i\left(r^{-1}\right)_{s}}{s}}\right)$

precisely matching (4.6).

\section{APPENDIX C: ACCUMULATION POINT IN TWIST}

In Sec. V, we gave a suggestive argument that the twist gap $2 t_{\text {gap }}$ cannot exceed $\frac{c-1}{16}$. However, there we assumed that the contribution of the lowest twist nonvacuum operator to the density of large spin states came from a finite number of low-twist operators. It is possible that the twist gap comes from an accumulation point in twist. In that case, it is not obvious that the contribution to the high-spin density of states has the $\bar{h}$ dependence in (5.4).

We start with the modular constraint (2.13) rewritten as

$$
\begin{aligned}
\sum_{j=0}^{\infty} & e^{-\beta j} \int_{0}^{\infty} d \bar{h} \rho_{j}(\bar{h}) \exp \left[-\bar{\beta}\left(\bar{h}-\frac{c-1}{24}\right)\right] \\
= & \sum_{j=0}^{\infty} e^{-\beta j} \int_{0}^{\infty} d \bar{h} \rho_{j}^{0}(\bar{h}) \exp \left[-\bar{\beta}\left(\bar{h}-\frac{c-1}{24}\right)\right] \\
& +\mathcal{O}\left(e^{\frac{4 \pi^{2}}{\beta}\left(\frac{c-1}{24}-t_{\mathrm{gap}}\right)}\right) .
\end{aligned}
$$

Recall that $\rho_{j}(\bar{h})$ is the physical density of states (2.9) and $\rho_{j}^{0}(\bar{h})$ is defined in (4.11). We perform an inverse Laplace transform in $\beta{ }^{20}$

$$
\begin{aligned}
\int_{0}^{\infty} & d \bar{h} \rho_{j}(\bar{h}) \exp \left[-\bar{\beta}\left(\bar{h}-\frac{c-1}{24}\right)\right] \\
= & \int_{0}^{\infty} d \bar{h} \rho_{j}^{0}(\bar{h}) \exp \left[-\bar{\beta}\left(\bar{h}-\frac{c-1}{24}\right)\right] \\
& +\mathcal{O}\left(e^{\left.4 \pi \sqrt{j\left(\frac{c-1}{24}-t_{\text {gap }}\right.}\right)}\right) .
\end{aligned}
$$

It is then tempting to equate $\rho_{j}(\bar{h})$ with $\rho_{j}^{0}(\bar{h})$, but the inverse Laplace transform with respect to $\bar{\beta}$ is only unique up to a measure zero set of $\mathbb{R}^{+}$. Nonetheless, two piecewise continuous functions with the same Laplace transforms

\footnotetext{
${ }^{20}$ Here and below we assume that the inverse Laplace transform of the error term in $(\mathrm{C} 1)$ with respect to $\beta$ is dominated by the inverse Laplace transform of $e^{\frac{4 \pi^{2}}{\beta}\left(\frac{c-1}{24}-t_{\text {gap }}\right)}$ for large $j$. To prove this requires a refinement of the argument in [19] with a finite twist gap (or irrationality) condition imposed.
}

agree on the subset of $\mathbb{R}^{+}$where they are both continuous (see, e.g., [30]). To utilize this uniqueness property, we consider the integrated spectral density for $\rho_{j}(\bar{h})$ defined as

$$
F_{j}(\bar{h})=\int_{0}^{\bar{h}} d \bar{h}^{\prime} \rho_{j}\left(\bar{h}^{\prime}\right),
$$

which is piecewise continuous. Similarly we define $F_{j}^{0}(\bar{h})$ for $\rho_{j}^{0}(\bar{h})$. By performing integration by parts on $(\mathrm{C} 2)$, we obtain

$$
\begin{aligned}
\int_{0}^{\infty} & d \bar{h} F_{j}(\bar{h}) \exp \left[-\bar{\beta}\left(\bar{h}-\frac{c-1}{24}\right)\right] \\
= & \int_{0}^{\infty} d \bar{h} F_{j}^{0}(\bar{h}) \exp \left[-\bar{\beta}\left(\bar{h}-\frac{c-1}{24}\right)\right] \\
& +\mathcal{O}\left(e^{4 \pi \sqrt{j\left(\frac{c-1}{24}-t_{\mathrm{gap}}\right)}}\right)
\end{aligned}
$$

where we used $\lim _{\bar{h} \rightarrow \infty} F_{j}(\bar{h}) e^{-\bar{\beta} \bar{h}}=0$ as evident from the usual Cardy formula. Next performing the inverse Laplace transform in $\bar{\beta}$, we obtain

$$
F_{j}(\bar{h})=F_{j}^{0}(\bar{h})+\mathcal{O}\left(e^{4 \pi \sqrt{j\left(\frac{c-1}{24}-t_{\mathrm{gap}}\right)}}\right),
$$

where the equality holds away from the discontinuities of the LHS. We define the function

$$
g_{j}(\bar{h}) \equiv F_{j}(\bar{h})-F_{j}^{0}(\bar{h}) .
$$

It satisfies the following two properties:

(1) $\lim _{j \rightarrow \infty} \frac{g_{j}(\bar{h})}{\exp \left[4 \pi \sqrt{j\left(\frac{c-1}{24}-t_{\text {ga }}\right)}\right]}=G(\bar{h})$ where $G(\bar{h})$ is some finite function of $\bar{h}$.

(2) $g_{j}(\bar{h})$ is finite at every $\bar{h}$, and in particular $\lim _{\bar{h} \rightarrow \frac{c-1}{24}} g_{j}(\bar{h})$ is finite.

The first property follows from (C5) while the second follows from the finiteness of the number of states up to twist $\bar{h}$ at a given spin $j$.

The question we are interested in is the large $j$ growth of $g_{j}(\bar{h})$ in the double limit (5.2). To make it more precise, let us choose any function $f(j)$ such that

$0<f(j)-\frac{c-1}{24}<\frac{1}{8 \pi^{2}} \exp \left[-2 \pi \sqrt{\frac{c-1}{24} j}\right], \quad j:$ odd

We would like to know the large $j$ behavior of $g_{j}(\bar{h}=f(j))$. In this limit, we have shown in Sec. V that

$$
F_{j}^{0}(\bar{h}) \sim-e^{\pi \sqrt{j^{\frac{c-1}{24}}}} .
$$

If we naively commute the two limits $j \rightarrow \infty$ and $\bar{h} \rightarrow \frac{c-1}{24}$, we would have claimed that $g_{j}(\bar{h}=f(j))$ grows no faster than $e^{4 \pi \sqrt{j\left(\frac{c-1}{24}-t_{\text {gap }}\right)}}$; i.e., 


$$
\lim _{j \rightarrow \infty} \frac{g_{j}(f(j))}{\exp \left[4 \pi \sqrt{j\left(\frac{c-1}{24}-t_{\text {gap }}\right)}\right]}
$$

is finite. If this were the case, then for the negativity of $F_{j}^{0}(\bar{h}) \sim-e^{\pi \sqrt{\frac{c-1}{24} j}}$ to be canceled by the growth $e^{4 \pi \sqrt{j\left(\frac{c-1}{24}-t_{\text {gap }}\right)}}$, the twist has to be no bigger than

$$
t_{\text {gap }} \leq\left(\frac{15}{16}\right)\left(\frac{c-1}{24}\right) .
$$

If it can be proven that (C9) not only is finite but also vanished as $\sqrt{\bar{h}-\frac{c-1}{24}}$ (as would be the case if there were a finite number of low-twist operators), we would recover the original claim of $t_{\text {gap }} \leq \frac{c-1}{32}$ in Sec. V. However, Eq. (C9) involves a double limit on both $\bar{h}$ and $j$, whose behavior does not follow from the two properties of $g_{j}(\bar{h})$ above. We leave a rigorous derivation of the large $j$ behavior of $g_{j}(f(j))$ for the future.

\section{APPENDIX D: MODULAR TRANSFORMATION OF VIRASORO CHARACTERS}

In this Appendix, we derive simplified formulas for the modular kernel of general $\operatorname{PSL}(2, \mathbb{Z})$ transformations for Virasoro characters at central charge $c>1$. See [31,32] for a nice review on this subject. We will mostly follow the conventions of [31] here and focus on the holomorphic side.

It is convenient to parametrize the CFT central charge as

$$
c=1+6 Q^{2}, \quad Q=b+\frac{1}{b},
$$

where $0<b<1$ for $c>25$ or $b$ is a complex phase with $|b|=1$ for $1<c \leq 25$, inspired by the Liouville theory. Similarly, we label Virasoro primaries of weight $h$ by the Liouville momenta

$$
\alpha \equiv \frac{Q+i p}{2}, \quad h=\alpha(Q-\alpha)
$$

such that for $p \in \mathbb{R}^{+}$, we have a nondegenerate primary with character and weight

$$
\chi_{p}(\tau)=\frac{q^{\frac{p^{2}}{4}}}{\eta(\tau)}, \quad h_{p}=\frac{Q^{2}+p^{2}}{4}
$$

For imaginary $p=i(m / b+n b)$ with $m, n \in \mathbb{N}$, we have a degenerate primary (which has a single null vector at level $m n$ for generic $b$ ) with character and weight

$$
\begin{aligned}
& \chi_{m, n}=\frac{q^{-\frac{(m / b-n b)^{2}}{4}}-q^{-\frac{(m / b+n b)^{2}}{4}}}{\eta(\tau)}, \\
& h_{m, n}=\frac{Q^{2}-(m / b+n b)^{2}}{4} .
\end{aligned}
$$

In particular, the vacuum is identified with the $m=n=1$ degenerate primary

$$
\chi_{\mathrm{vac}} \equiv \chi_{1,1}
$$

The $S$ transformation of the Virasoro characters are particularly simple in Liouville notation,

$$
\begin{aligned}
\chi_{p}(-1 / \tau) & =\int_{0}^{\infty} d p^{\prime} S_{p}{ }^{p^{\prime}} \chi_{p^{\prime}}, \quad S_{p}{ }^{\prime}=\sqrt{2} \cos \left(\pi p p^{\prime}\right), \\
\chi_{m, n}(-1 / \tau) & =\int_{0}^{\infty} d p^{\prime} S_{m, n}{ }^{p^{\prime}} \chi_{p^{\prime}}, \\
S_{m, n} p^{\prime} & =2 \sqrt{2} \sinh \left(\pi m p^{\prime} / b\right) \sinh \left(\pi n b p^{\prime}\right),
\end{aligned}
$$

while the $T$ transformation gives a phase

$$
\begin{aligned}
\chi_{p}(\tau+1) & =e^{\frac{\pi i}{2} p^{2}} e^{\frac{\pi i}{12}} \chi_{p}(\tau), \\
\chi_{m, n}(\tau+1) & =e^{-\frac{\pi i}{2}(m / b+n b)^{2}} e^{\frac{\pi i}{12}} \chi_{m, n}(\tau) .
\end{aligned}
$$

For a given element $\gamma \in \operatorname{PSL}(2, \mathbb{Z})$ generated by a sequence of $S$ and $T$ transformations, it is straightforward to compose the integrals and derive the modular kernel $\mathbf{K}_{\gamma} \cdot{ }^{21}$ In particular,

$$
\mathbf{K}_{S}(m, n ; p)=S_{m, n}{ }^{p}, \quad \mathbf{K}_{S}\left(p ; p^{\prime}\right)=S_{p}{ }^{\prime} .
$$

For illustration, let us look at the $S T^{n} S$ transformation (with $n \in \mathbb{N}$ )

$$
\gamma \cdot \tau=\frac{\tau}{1-n \tau}
$$

of the identity character

$$
\begin{aligned}
\chi_{\mathrm{vac}}(\gamma \cdot \tau)= & 2 e^{\frac{-n \pi i}{12}} \int_{\mathbb{R}^{+}} d p^{\prime} \sinh \left(\pi p^{\prime} / b\right) \sinh \left(\pi b p^{\prime}\right) e^{\frac{n \pi i p^{\prime 2}}{2}} \\
& \times \int_{\mathbb{R}} d p e^{i \pi p p^{\prime}} \chi_{p}(\tau) .
\end{aligned}
$$

We would like to perform the $p^{\prime}$ integral first but as it stands above, the integral diverges exponentially. We can get around this by shifting the contour of the $p$ integral in the

\footnotetext{
${ }^{21}$ Note that the modular crossing kernel $\mathbf{K}_{\gamma}$ here differs from $K_{\gamma}$ in the main text [e.g., (3.3)] by the choice of integration measure over Virasoro modules. For example, $K_{\gamma}\left(h_{p}\right)=$ $\frac{1}{\sqrt{h_{p}-\frac{c-1}{24}}} \mathbf{K}_{\gamma}(\mathrm{vac} ; p)$ for nondegenerate modules labeled by $p$.
} 
imaginary direction by $\Delta=1 / b+b+\epsilon$ for $\epsilon>0$ [31]

Since there are no poles in $p$, we obtain

$$
\begin{aligned}
\chi_{\mathrm{vac}}(\gamma \cdot \tau)= & 2 e^{\frac{-n \pi i}{12}} \int_{\mathbb{R}^{+}} d p^{\prime} \sinh \left(\pi p^{\prime} / b\right) \sinh \left(\pi b p^{\prime}\right) e^{\frac{n \pi i p^{\prime 2}}{2}} \\
& \times \int_{\mathbb{R}+i \Delta} d p e^{i \pi p p^{\prime}} \chi_{p}(\tau)
\end{aligned}
$$

and can now integrate over $p^{\prime}$ using the following identities that involve the (complement) error function $\operatorname{erfc}(z)$ or equivalently the Faddeeva function $w(i z)$ :

$$
\begin{aligned}
\int_{\mathbb{R}^{+}} d p^{\prime} e^{\pi p^{\prime}\left(\epsilon_{1} / b+\epsilon_{2} b\right)} e^{n \pi i p^{2} / 2} e^{i \pi p p^{\prime}} \\
=\frac{e^{\frac{\pi i}{4}}}{\sqrt{2 n}} e^{z\left(p, \epsilon_{1}, \epsilon_{2}\right)^{2}} \operatorname{erfc}\left(z\left(p, \epsilon_{1}, \epsilon_{2}\right)\right) \\
=\frac{e^{\frac{\pi i}{4}}}{\sqrt{2 n}} w\left(i z\left(p, \epsilon_{1}, \epsilon_{2}\right)\right),
\end{aligned}
$$

where

$$
\begin{aligned}
\operatorname{erfc}(z) & \equiv \frac{2}{\sqrt{\pi}} \int_{z}^{\infty} e^{-t^{2}} d t=1-\operatorname{erf}(z) \\
w(z) & =e^{-z^{2}} \operatorname{erfc}(-i z)
\end{aligned}
$$

with

$$
z\left(p, \epsilon_{1}, \epsilon_{2}\right)=e^{-3 \pi i / 4} \sqrt{\frac{\pi}{2 n}}\left(\epsilon_{1} / b+\epsilon_{2} b+i p\right) .
$$

Now using the fact that $w(i z)$ is an entire function ${ }^{22}$ and the exponential suppression from $\chi_{p}(\tau)$ in the $p$ integral, we can deform the $p$-integration contour back to $\mathbb{R}$ and (D11) becomes

$$
\begin{aligned}
\chi_{\mathrm{vac}}(\gamma \cdot \tau) & =\frac{e^{\frac{(3-n) \pi i}{12}}}{2 \sqrt{2 n}} \sum_{\epsilon_{1,2}= \pm} \int_{\mathbb{R}} d p w\left(i z\left(p, \epsilon_{1}, \epsilon_{2}\right)\right) \chi_{p}(\tau) \\
& =\frac{e^{\frac{(3-n) \pi i}{12}}}{\sqrt{2 n}} \int_{\mathbb{R}} d p\left(e^{z(p, 1,1)^{2}}-e^{z(p, 1,-1)^{2}}\right) \chi_{p}(\tau) \\
& =\frac{e^{\frac{(3-n) \pi i}{12}}}{\sqrt{2 n}} \int_{\mathbb{R}^{+}} d p\left(e^{\frac{\pi i}{2 n}(b+1 / b+i s)^{2}}-e^{\frac{\pi i}{2 n}(b-1 / b+i s)^{2}}+e^{\frac{\pi i}{2 n}(b+1 / b-i s)^{2}}-e^{\frac{\pi i}{2 n}(b-1 / b-i s)^{2}}\right) \chi_{p}(\tau) .
\end{aligned}
$$

In the second equality above, we have used $w(-z)=2 e^{-z^{2}}-w(z)$. We read off the modular kernel $\mathbf{K}_{S T^{n} S}(\operatorname{vac} ; p)$ from the last line of (D15) to be

$$
\begin{aligned}
\mathbf{K}_{S T^{n} S}(\operatorname{vac} ; p)= & \frac{2 e^{\frac{(3-n) \pi i}{12}}}{\sqrt{2 n}} e^{\frac{2 \pi i(c-1)}{24 n}} e^{\frac{-\pi i p^{2}}{2 n}}\left(\cosh \left(\frac{\pi p(b+1 / b)}{n}\right)\right. \\
& \left.-e^{\frac{-2 \pi i}{n}} \cosh \left(\frac{\pi p(b-1 / b)}{n}\right)\right) \cdot \quad \text { (D16) }
\end{aligned}
$$

As a check of (D16), note that in $\operatorname{PSL}(2, \mathbb{Z})$, we have the identity $S T S=T^{-1} S T^{-1}$. One can immediately write down the modular transformation of $\chi_{\mathrm{vac}}$ under $T^{-1} S T^{-1}$ using (D6) and (D7),

\footnotetext{
${ }^{22}$ For more properties of the Faddeeva function and related functions see Chapter 7 of [33].
}

$$
\begin{aligned}
\chi_{\mathrm{vac}}(\gamma \cdot \tau)= & 2 \sqrt{2} e^{\frac{2 \pi i}{12} e^{\frac{2 \pi i(c-1)}{24}}} \\
& \times \int_{\mathbb{R}^{+}} d p \sinh (\pi p / b) \sinh (\pi b p) e^{\frac{-\pi i p^{2}}{2}} \chi_{p}(\tau),
\end{aligned}
$$

and we see that this indeed matches (D16) when $n=1$.

Similarly, it is straightforward to derive the $S T^{n} S$ transformation of a nondegenerate Virasoro character labeled by $s \in \mathbb{R}^{+}$,

$$
\begin{aligned}
\chi_{s}(\gamma \cdot \tau) & =\frac{1}{2} e^{\frac{-n \pi i}{12}} \int_{\mathbb{R}} d p^{\prime} e^{i \pi s p^{\prime}} e^{\frac{n \pi i p^{2}}{2}} \int_{\mathbb{R}} d p e^{i \pi p p^{\prime}} \chi_{p}(\tau) \\
& =\frac{1}{\sqrt{2 n}} e^{\frac{\pi(3-n)}{12}} \int d p e^{-\frac{\pi i(p+s)^{2}}{2 n}} \chi_{p}(\tau),
\end{aligned}
$$

and thus

$$
\mathbf{K}_{S T^{n} S}(s ; p)=\frac{1}{\sqrt{2 n}} e^{\frac{\pi i(3-n)}{12}} e^{-\frac{\pi i(p+s)^{2}}{2 n}}
$$


[1] S. Collier, Y.-H. Lin, and X. Yin, Modular bootstrap revisited, J. High Energy Phys. 09 (2018) 061.

[2] J. L. Cardy, Operator content of two-dimensional conformally invariant theories, Nucl. Phys. B270, 186 (1986).

[3] A. L. Fitzpatrick, J. Kaplan, D. Poland, and D. SimmonsDuffin, The analytic bootstrap and AdS superhorizon locality, J. High Energy Phys. 12 (2013) 004.

[4] Z. Komargodski and A. Zhiboedov, Convexity and liberation at large spin, J. High Energy Phys. 11 (2013) 140.

[5] S. Hellerman, A Universal inequality for CFT and quantum gravity, J. High Energy Phys. 08 (2011) 130.

[6] Y. Kusuki, Light cone bootstrap in general 2D CFTs and entanglement from light cone singularity, J. High Energy Phys. 01 (2019) 025.

[7] S. Collier, Y. Gobeil, H. Maxfield, and E. Perlmutter, Quantum Regge trajectories and the Virasoro analytic bootstrap, J. High Energy Phys. 05 (2019) 212.

[8] Y. Kusuki and M. Miyaji, Entanglement entropy, OTOC and bootstrap in 2D CFTs from Regge and light cone limits of multi-point conformal block, J. High Energy Phys. 08 (2019) 063.

[9] J. M. Maldacena, The large N limit of superconformal field theories and supergravity, Int. J. Theor. Phys. 38, 1113 (1999); Adv. Theor. Math. Phys. 2, 231 (1998).

[10] P. Saad, S. H. Shenker, and D. Stanford, JT gravity as a matrix integral, arXiv:1903.11115.

[11] H. Ooguri and C. Vafa, Non-supersymmetric AdS and the Swampland, Adv. Theor. Math. Phys. 21, 1787 (2017).

[12] A. Maloney and E. Witten, Quantum gravity partition functions in three dimensions, J. High Energy Phys. 02 (2010) 029.

[13] C. A. Keller and A. Maloney, Poincare series, 3D gravity and CFT spectroscopy, J. High Energy Phys. 02 (2015) 080.

[14] R. Dijkgraaf, J. M. Maldacena, G. W. Moore, and E. P. Verlinde, A black hole Farey tail, arXiv:hep-th/0005003.

[15] J. M. Maldacena and A. Strominger, AdS(3) black holes and a stringy exclusion principle, J. High Energy Phys. 12 (1998) 005.

[16] A. Maloney, H. Maxfield, and G. S. Ng, A conformal block Farey tail, J. High Energy Phys. 06 (2017) 117.

[17] N. Afkhami-Jeddi, K. Colville, T. Hartman, A. Maloney, and E. Perlmutter, Constraints on higher spin $\mathrm{CFT}_{2}$, J. High Energy Phys. 05 (2018) 092.
[18] A. B. Zamolodchikov and A. B. Zamolodchikov, Liouville field theory on a pseudosphere, arXiv:hep-th/0101152.

[19] B. Mukhametzhanov and A. Zhiboedov, Modular invariance, Tauberian theorems, and microcanonical entropy, arXiv: 1904.06359.

[20] S. Ganguly and S. Pal, Bounds on density of states and spectral gap in $\mathrm{CFT}_{2}$, arXiv:1905.12636.

[21] D. Das, S. Datta, and S. Pal, Charged structure constants from modularity, J. High Energy Phys. 11 (2017) 183.

[22] H. Maxfield, Quantum corrections to the BTZ black hole extremality bound from the conformal bootstrap, arXiv: 1906.04416.

[23] D. Mazac, Analytic bounds and emergence of $\mathrm{AdS}_{2}$ physics from the conformal bootstrap, J. High Energy Phys. 04 (2017) 146.

[24] I. Esterlis, A. L. Fitzpatrick, and D. Ramirez, Closure of the operator product expansion in the non-unitary bootstrap, J. High Energy Phys. 11 (2016) 030.

[25] S. Collier, P. Kravchuk, Y.-H. Lin, and X. Yin, Bootstrapping the spectral function: On the uniqueness of Liouville and the Universality of BTZ, J. High Energy Phys. 09 (2018) 150.

[26] A. Strominger, Black hole entropy from near horizon microstates, J. High Energy Phys. 02 (1998) 009.

[27] E. Witten, Three-dimensional gravity revisited, arXiv:0706 .3359 .

[28] F. Olver and W. Rheinbolt, Asymptotics and Special Functions (Elsevier Science, San Diego, 2014).

[29] G. H. Hardy, Divergent Series (Oxford University Press, Clarendon Press, Oxford, 1967).

[30] G. Doetsch and W. Nader, Introduction to the Theory and Application of the Laplace Transformation (Springer, Berlin Heidelberg, 2012).

[31] C. Jego and J. Troost, Notes on the Verlinde formula in nonrational conformal field theories, Phys. Rev. D 74, 106002 (2006).

[32] S. Ribault, Minimal lectures on two-dimensional conformal field theory, SciPost Phys. Lect. Notes 1, 2016 (2018).

[33] F. W. J. Olver, A. B. Olde Daalhuis, D. W. Lozier, B. I. Schneider, R. F. Boisvert, C. W. Clark, B. R. Miller, and B. V. Saunders, NIST Digital Library of Mathematical Functions, http://dlmf.nist.gov/, Release 1.0.22 of 201903-15. 